\title{
Sensitivity of the SHiP experiment to dark photons decaying to a pair of charged particles
}

\author{
SHiP Collaboration ${ }^{\star}$
}

Received: 11 November 2020 / Accepted: 10 May 2021 / Published online: 25 May 2021

(C) The Author(s) 2021

\begin{abstract}
Dark photons are hypothetical massive vector particles that could mix with ordinary photons. The simplest theoretical model is fully characterised by only two parameters: the mass of the dark photon $\mathrm{m}_{\gamma} \mathrm{D}$ and its mixing parameter with the photon, $\varepsilon$. The sensitivity of the SHiP detector is reviewed for dark photons in the mass range between 0.002 and $10 \mathrm{GeV}$. Different production mechanisms are simulated, with the dark photons decaying to pairs of visible fermions, including both leptons and quarks. Exclusion contours are presented and compared with those of past experiments. The SHiP detector is expected to have a unique sensitivity for $\mathrm{m}_{\gamma^{\mathrm{D}}}$ ranging between 0.8 and $3.3_{-0.5}^{+0.2} \mathrm{GeV}$, and $\varepsilon^{2}$ ranging between $10^{-11}$ and $10^{-17}$.
\end{abstract}

\section{Introduction}

The CERN beam facility located near Geneva, Switzerland, comprises several particle accelerators among which the Super Proton Synchrotron (SPS) and the Large Hadron Collider (LHC) [1]. The SPS is an essential part of the accelerator chain delivering $400 \mathrm{GeV}$ proton beams to the $\mathrm{LHC}$ but also to fixed-target experiments. The LHC is planned to be upgraded into a high-luminosity machine starting operation around 2026 with the HL-LHC program [2]. In parallel to the high-energy frontier probed by the LHC, a complementary way of exploring the parameter space of potential new physics is through the "intensity frontier". The SPS physics programme is hence proposed to be further extended via the construction of a beam dump facility (BDF) [3]. The BDF foresees the full exploitation of the SPS accelerator, which with its present performance could allow the delivery of up to $4 \times 10^{19}$ protons on target per year, while respecting the beam requirements of the HL-LHC and maintaining the operation of the existing SPS beam facilities.

By probing lower-energy scenarios with high-intensity beams, the aim is to identify whether new physics could be

^e-mail: a.magnan@imperial.ac.uk hidden from sight due to weak connections through portals instead of direct interactions with the known particles, with the new particles belonging to a hidden sector. The simplest renormalisable extensions of the standard model (SM) are possible through three types of portals [4,5], involving either a scalar (e.g. dark Higgs boson [6,7]), a vector (e.g. dark photon $[8,9]$ ) or fermions (e.g. heavy neutral leptons [10]). The LHC experiments have already derived strong constraints on short-lived high-mass mediators [11-14]. Scenarios with long-lived mediators with relatively low masses however remain largely unexplored. The SHiP (Search for Hidden Particles) experiment [15] has been proposed in 2013 [16] and is designed to look for particles which would decay in the range $50-120 \mathrm{~m}$ from their production vertices. The sensitivity of the SHiP detector to heavy neutral leptons has been investigated in Ref. [17]. This article is dedicated to studying the sensitivity of the SHiP detector to dark photons.

After describing briefly the SHiP detector and its simulation in Sect. 2, the model considered for the dark photon production and decay is reviewed in Sect. 3. The sensitivity of the SHiP detector in the minimal dark photon model with decays to charged particles is given in Sect. 4 for the three production modes studied. Finally Sect. 5 provides a conclusion.

\section{The SHiP detector and simulation}

SHiP [15] is a new general purpose fixed-target experiment intended to exploit the proposed BDF to search for particles present in hidden portal models. The $400 \mathrm{GeV}$ proton beam extracted from the SPS will be dumped on a high density target with the aim of accumulating $2 \times 10^{20}$ protons on target during 5 years of operation. A dedicated detector, based on a long vacuum tank followed by a spectrometer and particle identification detectors, will allow probing a variety of models with light long-lived exotic particles and masses below $\mathcal{O}(10) \mathrm{GeV}$. A critical component of SHiP is the muon shield, which deflects the high flux of muons 
Fig. 1 Positions of the vessel and tracking stations along the beam axis $(\mathrm{z})$. The vessel dimensions $\delta x_{\text {phys }}$ and $\delta y_{\text {phys }}$ represent the upstream and downstream physics acceptance in the plane transverse to the beam axis
SHiP Spectrometer Tracker

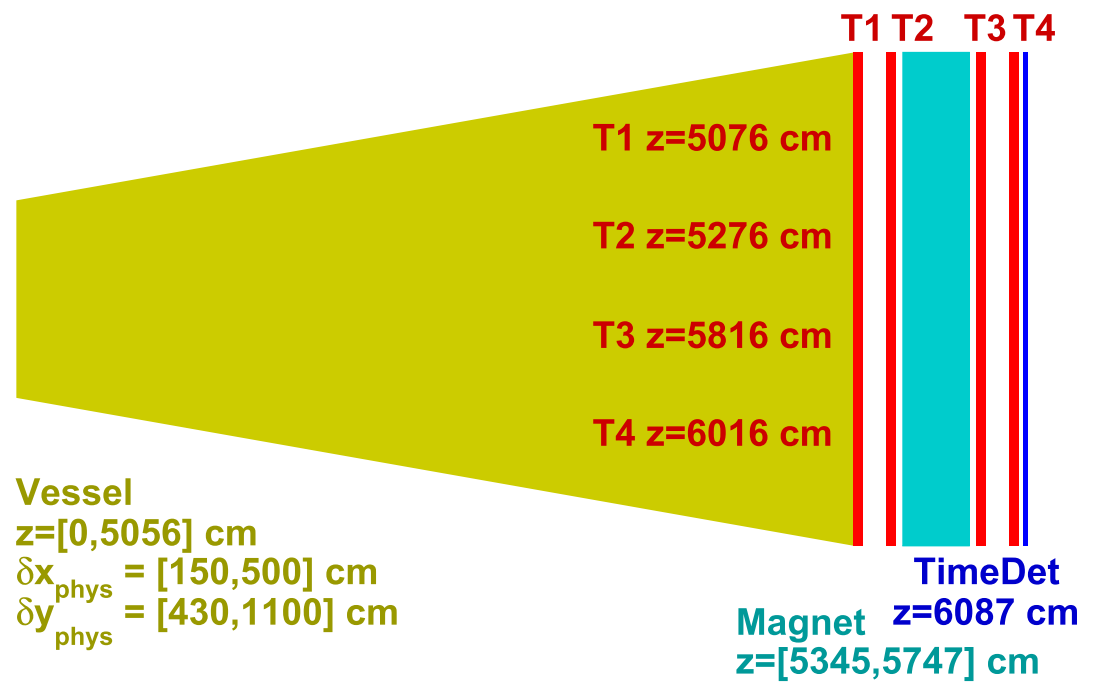

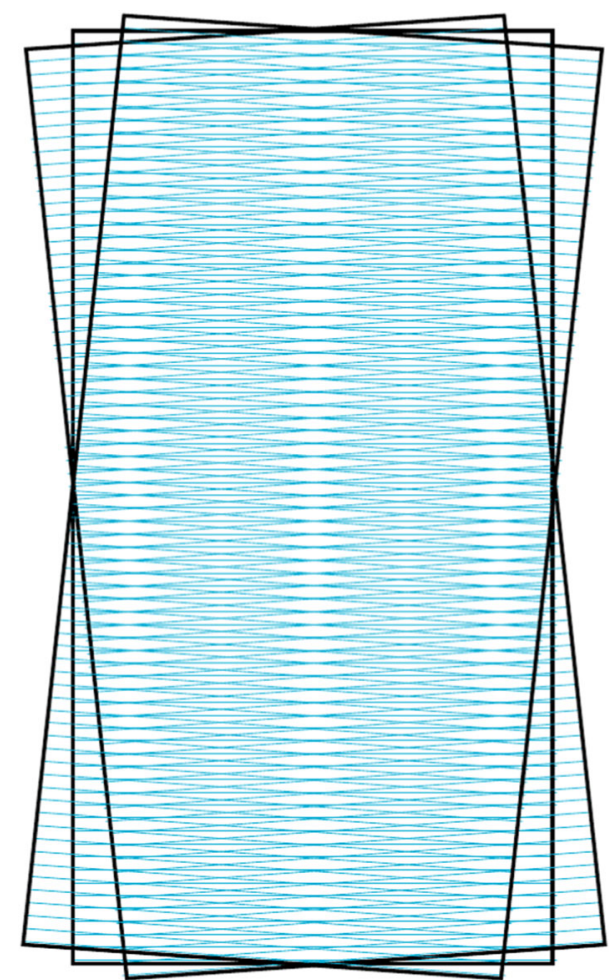

Fig. 2 Schematic drawing of the three "views" that compose each straw chamber

produced in the target $[18,19]$, that would represent a serious background in the search for hidden-sector particles. The detector is designed to fully reconstruct the exclusive decays of hidden particles and to reject the background down to below 0.1 events in the sample of $2 \times 10^{20}$ protons on target [20].

The detector consists of a large magnetic spectrometer located downstream of a $50 \mathrm{~m}$-long and up to $5 \times 11 \mathrm{~m}$ - wide cone-shaped decay volume [21]. To suppress the background from neutrinos interacting in the fiducial volume, the decay volume is maintained under a vacuum. The spectrometer tracker is designed to accurately reconstruct the decay vertex, mass and impact parameter of the decaying particle. A set of calorimeters followed by muon chambers provide identification of electrons, photons, muons and charged hadrons. A dedicated timing detector measures the coincidence of the decay products, which allows the rejection of combinatorial backgrounds. The decay volume is surrounded by background taggers to tag neutrino and muon inelastic scattering in the surrounding structures, which may produce long-lived $\mathrm{SM} \mathrm{V}^{0}$ particles, such as $\mathrm{K}_{\mathrm{L}}$, that have topologies similar to the expected signals.

The spectrometer tracker is a crucial component in the reconstruction of the charged particles produced by the decay of dark photons. The baseline layout consists of four tracking stations (T1-T4) symmetrically arranged around a dipole magnet as shown in Fig. 1. The transverse size of the tracker stations matches the size of the magnet. Each station consists of 9072 straw tubes which are arranged in four views (Y-U-V-Y), as shown in Fig. 2. The Y view has straws horizontally aligned. The $\mathrm{U}$ and $\mathrm{V}$ views are rotated by an angle of $\theta_{\text {stereo }}= \pm 5^{\circ}$. The $\mathrm{x}$ coordinate is hence measured with an accuracy of $1 / \sin \left(\theta_{\text {stereo }}\right)$, directly impacting the measurement of the decay vertex, of the opening angle of the daughter particles (which enters the invariant mass), and of the impact parameter at the production target. In order to provide good spatial resolution and minimise the contribution from multiple scattering, the straw tubes are made of thin polyethylene terephthalate (PET). More detail about the initial design of the straw detector can be found in Refs. [20,22]. The pattern recognition algorithms applied to the hits on the straw spec- 
trometer are described in Ref. [23], and the algorithms for particle identification are presented in Ref. [24].

In the simulation, proton fixed-target collisions are generated by PYTHIA 8.2 [25], inelastic neutrino interactions by GENIE [26] and inelastic muon interactions by PYTHIA 6 [27]. The heavy-flavour cascade production is also taken into account [28]. The SHiP detector response is simulated in the GEANT4 [29] framework. The simulation is done within FairShip, which is based on the FairRoot framework [30].

\section{Dark photon production and decay}

The minimal dark photon model contains an additional $\mathcal{U}(1)$ gauge group to the $\mathrm{SM}, \mathrm{A}_{\mu}^{\prime}$, whose vector gauge boson is called the dark photon $\gamma^{\mathrm{D}}$. A kinetic mixing term between the dark photon with field strength $F_{\mu \nu}^{\prime}$ and the SM $\mathcal{U}(1)$ gauge bosons with field strength $F_{Y}^{\mu \nu}$ is allowed [8], with a reduced strength parameterised by a coupling $\varepsilon$, also called the kinetic mixing parameter. The corresponding terms in the Lagrangian can hence be written as:

$\mathcal{L}=\mathcal{L}_{\mathrm{SM}}-\frac{1}{4}\left(F_{\mu \nu}^{\prime}\right)^{2}-\frac{\varepsilon}{2} F_{\mu \nu}^{\prime} F_{Y}^{\mu \nu}+\frac{1}{2} m_{\gamma^{\mathrm{D}}}^{2}\left(A_{\mu}^{\prime}\right)^{2}$.

In its simplest form, the knowledge of the mass of the dark photon $m_{\gamma}$ D and the kinetic mixing parameter $\varepsilon$ is enough to characterise the model and calculate production cross section and decay properties.

Three different mechanisms are possible for the production of such new particles at a fixed-target experiment. All of them are studied in this paper.

The initial $400 \mathrm{GeV}$ proton beam interacts with the nucleons from the target material, producing mesons. For meson decay channels involving photons, the photon can mix with the dark photon, as described in Sect. 3.1. This mode is opened only for dark photon masses below $0.9 \mathrm{GeV}$, as for mesons with masses above this threshold the main decay channels do not involve photons anymore.

The proton-nucleon interaction could also lead to the radiation of a dark photon via a bremsstrahlung process, as described in Sect. 3.2. This mode is heavily suppressed when the dark photon mass exceeds that of the proton, and remains relevant only for masses below $\simeq 2 \mathrm{GeV}$.

The third production mode is via a Drell-Yan like process in Quantum Chromodynamic (QCD), i.e. quark-antiquark annihilation into the dark photon, as described in Sect. 3.3. This process is relevant for dark photon masses in the range $\mathcal{O}(1-10) \mathrm{GeV}$. Using the parton model with a factorisation scale below the GeV scale cannot give sensible results, as expected from the range of validity of parton distribution functions, and hence this region of the parameter space has not been considered for this production mechanism.
Table 1 Meson decay channels considered for the $\gamma^{\mathrm{D}}$ production. The last column shows the average number of mesons expected per protonproton interaction

\begin{tabular}{llll}
\hline$m_{\gamma^{\mathrm{D}}}(\mathrm{GeV})$ & Meson & $\operatorname{Br}(\gamma+X)[25]$ & $\mathrm{n}_{\text {meson }} / \mathrm{pp}$ \\
\hline $0-0.135$ & $\pi^{0} \rightarrow \gamma^{\mathrm{D}} \gamma$ & 0.98799 & $6.147 \pm 0.003$ \\
$0-0.548$ & $\eta \rightarrow \gamma^{\mathrm{D}} \gamma$ & 0.3931181 & $0.703 \pm 0.008$ \\
$0-0.648$ & $\omega \rightarrow \gamma^{\mathrm{D}} \pi^{0}$ & 0.0834941 & $0.825 \pm 0.009$ \\
$0-0.958$ & $\eta^{\prime} \rightarrow \gamma^{\mathrm{D}} \gamma$ & 0.0219297 & $0.079 \pm 0.003$ \\
\hline
\end{tabular}

In this paper, the assumption is made that only the initial proton interacts. In reality, similar interactions could also happen with protons or mesons coming from cascade decays happening in the target material. For electromagnetic processes (electron bremsstrahlung of photons mixing with the dark photon), it has been shown in Ref. [31] that their contribution is negligible compared to the main production mechanisms described above. The study however remains to be done for hadronic interactions in the cascade decays, and will be the subject of future work. Hence the expectations presented here are conservative and the sensitivity could be improved in the future when this contribution is added.

As a final state, the dark photon decay to pairs of leptons or quarks as described in Sect. 3.4 is considered.

\subsection{Production in meson decay}

The PYTHIA 8.2 [25] Monte Carlo (MC) generator is used to produce inclusive QCD events in proton-proton ( $\mathrm{p}-\mathrm{p}$ ) collisions, through all available non-diffractive processes. Diffractive processes are less important in meson production, expected to decrease the number of mesons produced by about $15 \%$ according to PYTHIA simulation. Because the diffractive processes also suffer from larger theoretical uncertainties, they have not been considered. The leadingorder (LO) NNPDF2.3 PDF set [32] has been used with the default Monash 2013 tune [33], and the strong coupling constant set to $\alpha_{\mathrm{s}}=0.13$. One proton beam momentum is set to $400 \mathrm{GeV}$ and the other to 0 (protons or neutrons from the fixed-target material). The mesons that are produced are then used as sources of dark photons, if they have decay channels to photons and their decay to a dark photon of mass $m_{\gamma}$ D is kinematically allowed. Four processes are found dominant (with other contributions neglected) and shown in Table 1. The decay tables of these four mesons are reset to having only one decay channel allowed with $100 \%$ branching ratio $\left(\pi^{0} \rightarrow \gamma \gamma, \eta \rightarrow \gamma \gamma, \omega \rightarrow \pi^{0} \gamma, \eta^{\prime} \rightarrow \gamma \gamma\right)$. All relevant processes are then added together.

The branching ratios of the mesons to these new decay channels are functions of the $m_{\gamma}$ D, the kinetic mixing parameter $\varepsilon$, the meson type, pseudo-scalar or vector, and the meson mass $[4,31,34]$. For pseudo-scalar mesons $\mathcal{P}\left(\pi^{0}, \eta^{0}\right.$ and $\left.\eta^{\prime}\right)$, 
the branching ratio to $\gamma^{\mathrm{D}} \gamma$ is given by:

$\operatorname{Br}\left(\mathcal{P} \rightarrow \gamma^{\mathrm{D}} \gamma\right) \simeq 2 \varepsilon^{2}\left(1-\frac{m_{\gamma^{\mathrm{D}}}^{2}}{m_{\mathcal{P}}^{2}}\right)^{3} \operatorname{Br}(\mathcal{P} \rightarrow \gamma \gamma)$

For vector mesons $\mathcal{V}(\omega)$, the branching ratio to a $\gamma^{\mathrm{D}}$ and a pseudo-scalar meson $\mathcal{P}$ is given by:

$$
\begin{aligned}
& \operatorname{Br}\left(\mathcal{V} \rightarrow \mathcal{P} \gamma^{\mathrm{D}}\right) \simeq \varepsilon^{2} \times \operatorname{Br}(\mathcal{V} \rightarrow \mathcal{P} \gamma) \\
& \quad \times \frac{\left[\left(m_{\gamma^{\mathrm{D}}}^{2}-\left(m_{\mathcal{V}}+m_{\mathcal{P}}\right)^{2}\right)\left(m_{\gamma^{\mathrm{D}}}^{2}-\left(m_{\mathcal{V}}-m_{\mathcal{P}}\right)^{2}\right)\right]^{3 / 2}}{\left(m_{\mathcal{V}}^{2}-m_{\mathcal{P}}^{2}\right)^{3}}
\end{aligned}
$$

For the branching ratios of the mesons to $\gamma \gamma$ or $\gamma \pi^{0}$, the same values as implemented in PYTHIA 8.2 are used. The average number of mesons produced per pp interaction is shown for each meson type in the last column of Table 1, from nondiffractive pp collisions simulated with PYTHIA 8.2 [25], with its associated statistical uncertainty. For inclusive pp collisions (adding also elastic and diffractive processes), the average meson multiplicities predicted by Pythia are found to be in good agreement with existing measurements [35].

The cross section for the production of dark photons via meson decays produced in non-diffractive primary interactions of the proton beam is then computed as:

$$
\begin{aligned}
\sigma_{\text {meson }}= & \sigma_{\text {SHiP }}^{\text {inel }} \times \sum_{\text {mesons }} \Theta\left(m_{\text {meson }}-m_{\gamma^{\mathrm{D}}}\right) \\
& \times n_{\text {meson }} / \mathrm{pp} \times \operatorname{Br}\left(\text { meson } \rightarrow \gamma^{\mathrm{D}}+X\right),
\end{aligned}
$$

using Eqs. (2) and (3) and values reported in Table 1. The $\Theta\left(m_{\text {meson }}-m_{\gamma^{\mathrm{D}}}\right)$ factor is a step function ensuring that only the mesons in the accessible mass range are considered. To take into account the fact that the nucleon is bound in the target, and not free as assumed by PYTHIA in our simulation of this process, the total normalisation is taken using the inelastic proton-nucleon cross section corresponding to the SHiP target, $\sigma_{\text {SHiP }}^{\text {inel }}$ (see Sect. 4). In terms of the approximate scaling for hadron-nucleus interactions of $\sigma_{p A} / \sigma_{p p} \simeq A^{\alpha}$, this corresponds to $\alpha \simeq 0.71$. The cross section is proportional to $\varepsilon^{2}$, from the dependency of $\operatorname{Br}$ (meson $\rightarrow \gamma^{\mathrm{D}}+X$ ) in Eqs. (2) and (3).

\subsection{Production in proton bremsstrahlung}

In analogy with ordinary photon bremsstrahlung of scattering protons, the same process is used for dark photon production by scattering of the incoming $400 \mathrm{GeV}$ proton beam on the target protons. Following Refs. [31,36], the differential $\gamma^{\mathrm{D}}$ production rate can be expressed as:

$$
\begin{aligned}
\frac{\mathrm{d}^{2} N}{\mathrm{~d} z \mathrm{~d} p_{\perp}^{2}} & =\frac{\sigma_{p p}\left(s^{\prime}\right)}{\sigma_{p p}(s)} w_{b a}\left(z, p_{\perp}^{2}\right), \\
w_{b a}\left(z, p_{\perp}^{2}\right) & =\frac{\varepsilon^{2} \alpha_{\mathrm{QED}}}{2 \pi H}\left[\frac{1+(1-z)^{2}}{z}\right.
\end{aligned}
$$

$$
\begin{aligned}
& -2 z(1-z)\left(\frac{2 m_{p}^{2}+m_{\gamma^{\mathrm{D}}}^{2}}{H}-z^{2} \frac{2 m_{p}^{4}}{H^{2}}\right) \\
& +2 z(1-z)\left(1+(1-z)^{2}\right) \frac{m_{p}^{2} m_{\gamma^{\mathrm{D}}}^{2}}{H^{2}} \\
& \left.+2 z(1-z)^{2} \frac{m_{\gamma^{\mathrm{D}}}^{4}}{H^{2}}\right],
\end{aligned}
$$

where $\sigma_{p p}\left(s / s^{\prime}\right)$ are the total proton-proton cross sections evaluated for the incoming/outgoing proton energy scales, $m_{p}$ is the proton mass (set to $m_{p}=0.938272 \mathrm{GeV}$ [37]), $P$ and $E_{p}$ are the proton beam initial momentum and energy respectively, $p$ and $E_{\gamma}$ D are the momentum and energy of the generated dark photon respectively, $p_{\perp}$ and $p_{\|}$are the components of the $\gamma^{\mathrm{D}}$ momentum orthogonal and parallel to the direction of the incoming proton respectively, $z$ is the fraction of the proton momentum carried away by the dark photon in the beam direction, $\alpha_{\mathrm{QED}}$ is the fine structure constant of Quantum Electro Dynamic (QED), set to $1 / 137, s^{\prime}=2 m_{p}\left(E_{p}-E_{\gamma^{\mathrm{D}}}\right), s=2 m_{p} E_{p}$ and $H\left(p_{\perp}^{2}, z\right)=$ $p_{\perp}^{2}+(1-z) m_{\gamma^{\mathrm{D}}}^{2}+z^{2} m_{p}^{2}$.

In this formulation, the nuclear effects from having bound rather than free protons in the target material cancel in the ratio $\frac{\sigma_{p p}\left(s^{\prime}\right)}{\sigma_{p p}(s)}$.

However, the above formula does not take into account possible QCD contributions when the mass of the emitted $\gamma^{\mathrm{D}}$ exceeds that of the proton, and the bremsstrahlung process starts to depend on the internal partons. It does not take into account the possibility of enhancement in the cross section due to nuclear resonances in the so-called vector meson dominance (VMD) model either. In consequence, two independent approaches are followed, leading to two different estimates of the final cross section.

In the first approach, when the mass of the dark photon is larger than $1 \mathrm{GeV}$, the standard dipole form factor [38] is included in the proton- $\gamma^{\mathrm{D}}$ vertex, leading to a penalty factor that models the strong suppression of the bremsstrahlung production:

$\operatorname{penalty}\left(m_{\gamma^{\mathrm{D}}}\right)=\left(\frac{m_{\gamma^{\mathrm{D}}}^{2}}{0.71 \mathrm{GeV}^{2}}\right)^{-4}$ for $m_{\gamma^{\mathrm{D}}}^{2}>0.71 \mathrm{GeV}^{2}$.

According to Ref. [31], this form factor is conservative and probably underestimates the rates. The direct partonparton QCD production will dominate above $1.5 \mathrm{GeV}$ and is described in Sect. 3.3.

In the second approach, the VMD form factor taken from Refs. $[39,40]$ is used, leading to an enhancement of the cross section by a factor $10^{4}$ around the $\rho$ and $\omega$ meson mass of $0.8 \mathrm{GeV}$, and still up to a factor 10 in the tail due to also con- 
sidering resonances of masses 1.25 and $1.45 \mathrm{GeV}$ following the description in Ref. [40].

The total p-p cross section $\sigma_{p p}(s)$ is taken from experimental data:

$\sigma_{p p}(s)=Z+B \log ^{2}\left(\frac{s}{s_{0}}\right)+Y_{1}\left(\frac{s_{1}}{s}\right)^{\eta_{1}}-Y_{2}\left(\frac{s_{1}}{s}\right)^{\eta_{2}}$,

where $Z=35.45 \mathrm{mb}, B=0.308 \mathrm{mb}, Y_{1}=42.53 \mathrm{mb}$, $Y_{2}=33.34 \mathrm{mb}, \sqrt{s_{0}}=5.38 \mathrm{GeV}, \sqrt{s_{1}}=1 \mathrm{GeV}, \eta_{1}=0.458$ and $\eta_{2}=0.545$ [41]. This formulation has been compared to the latest parameterisation from Ref. [37], and found to be almost identical for the momentum range of interest here.

Reformulating Eq. (5) as a function of the $\gamma^{\mathrm{D}}$ angle $\theta$ to the beam line and its total momentum p, a two-dimensional normalised probability density function (PDF) $f(p, \theta)$ is extracted, and shown in Fig. 3 for two representative choices of $m_{\gamma}$. Note that due to the simple dependency of the production rate scaling as $\varepsilon^{2}$, the normalised PDF is independent of $\varepsilon$. The dark photons are generated with maximum probability on each side of the beam axis ( $\theta$ close to 0$)$ with a factor of 5 more chance to have $p<100 \mathrm{GeV}$ compared to $p>200 \mathrm{GeV}$, for the low masses, and increased probability to have high momentum as the mass increases.

Events are generated using a PYTHIA 8 particle gun with the $\gamma^{\mathrm{D}}$ as particle, randomly choosing the $\gamma^{\mathrm{D}}(\mathrm{p}, \theta)$ values according to the normalised 2D PDF $f(p, \theta)$, extracted for each $m_{\gamma}$ D point studied.

The integral of $\frac{\mathrm{d}^{2} N}{\mathrm{~d} p \mathrm{~d} \theta} \times \mathrm{FF}$, with FF the penalty dipole form factor or the VMD form factor, in the range of momenta and solid angle kinematically allowed, provides an estimate of the dark photon production rate per pp interaction through proton bremsstrahlung, scaling as $\varepsilon^{2}$. The production cross sections using the dipole form factor and VMD form factor methods are expressed by:

$\sigma_{\text {pbrem }}=\sigma_{\mathrm{SHiP}}^{\text {inel }} \times \int_{p_{\min }}^{p_{\max }} \int_{\theta=-\theta_{\max }}^{\theta_{\max }} \mathrm{FF} \times \frac{\mathrm{d}^{2} N}{\mathrm{~d} p \mathrm{~d} \theta} \mathrm{d} \theta \mathrm{d} p$,

and shown in Fig. 4. The conditions of validity of the approximation used to derive Eq. $(5)[42,43]$ require a lower momentum bound for the $\gamma^{\mathrm{D}}$ at $p_{\min }=0.1 P_{\mathrm{p}}$ [36], and an upper bound at $p_{\max }=0.9 P_{\mathrm{p}}$, as well as an upper bound on $p_{\perp}<4 \mathrm{GeV}$, giving $\theta_{\max } \simeq 0.1 \mathrm{rad}$.

\subsection{Drell-Yan production}

For production of the dark photon in parton-parton scattering, the generic implementation of a resonance that couples both to SM fermion pairs and hidden particles is used, as implemented in PYTHIA 8.2 under the "HiddenValley" $\mathrm{Z}$ ' model [44]. A cross-check has been done that similar kinematic distributions for the dark photons are found using
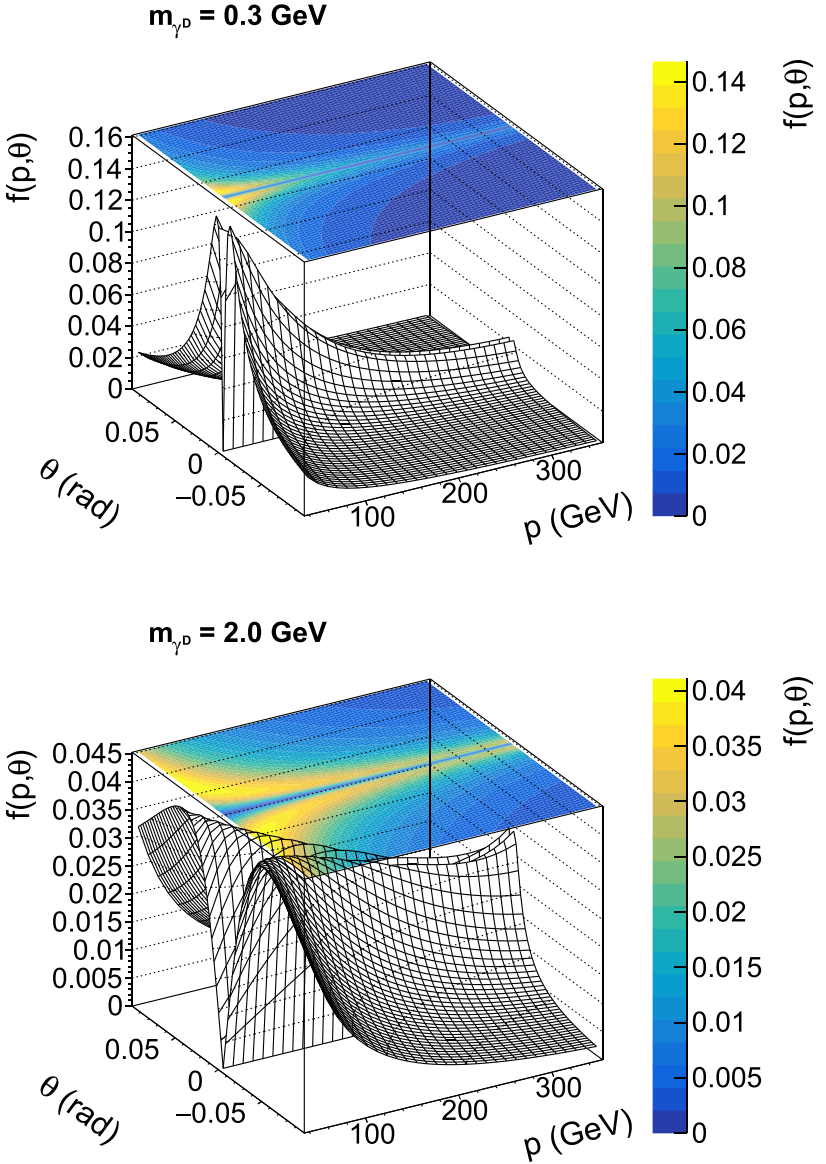

Fig. 3 Normalised probability density function of producing a dark photon with angle $\theta$ and momentum $p$ through proton bremsstrahlung, for two representative examples of $m_{\gamma^{\mathrm{D}}}: 0.3 \mathrm{GeV}$ (top) and $2 \mathrm{GeV}$ (bottom)

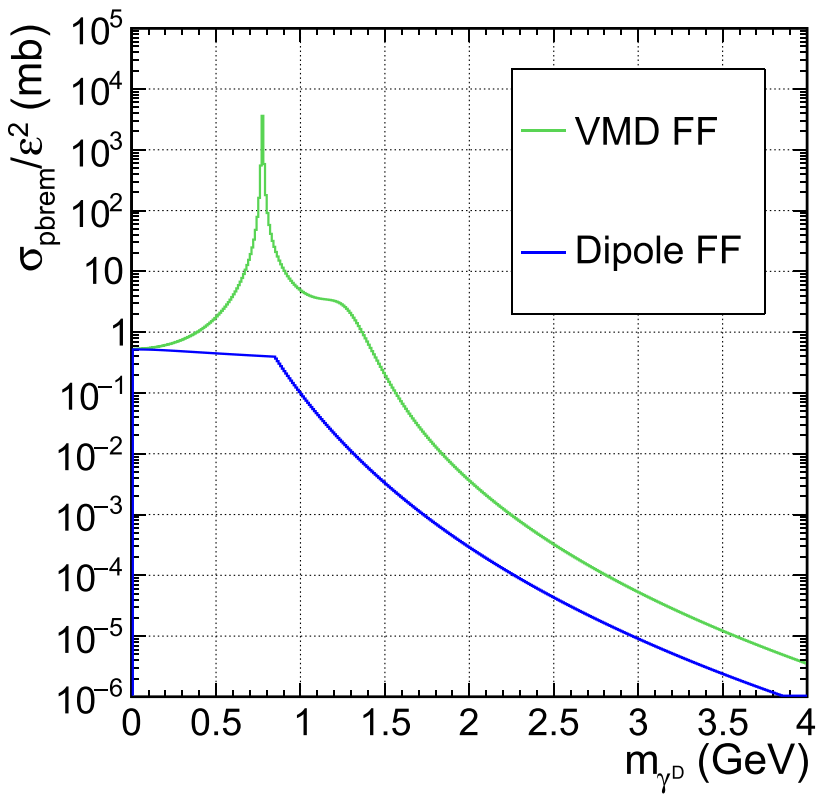

Fig. 4 Proton bremsstrahlung production cross section as a function of $m_{\gamma^{\mathrm{D}}}$ 
another Z' implementation in PYTHIA from the "New Gauge Bosons" class of processes [45].

The dark photons are generated in the mass range $1.4<$ $m_{\gamma^{\mathrm{D}}}<10 \mathrm{GeV}$. Below $1.4 \mathrm{GeV}$ one leaves the domain of perturbative QCD and the parton model cannot be used anymore.

The LO cross section given by PYTHIA when the new particle has the properties of the dark photon is shown in Fig. 5. The nuclear effects are neglected and the parton-parton cross section from PYTHIA is used without modification. Like for the meson and proton bremsstrahlung processes, it is found to scale as $\varepsilon^{2}$. The LO NNPDF2.3 PDF set [32] has been used with the default Monash 2013 tune [33], and the strong coupling constant set to $\alpha_{\mathrm{s}}=0.13$. An empirical function is extracted to parameterise the cross section (in $\mathrm{mb}$ ) as a function of the $\gamma^{\mathrm{D}}$ mass (in $\mathrm{GeV}$ ) in a continuous way, described in Eq. (9). The impact of several sources of theoretical uncertainties (PDF choice [46], QCD scales, $\alpha_{\mathrm{s}}$ ) are studied and shown in Fig. 5 (see also Sect. 4.4). The impact of nuclear effects is checked using the nuclear modification factors available in PYTHIA, with the most recent nuclear PDF set EPPS16 [47], using the two atomic masses $(\mathcal{A}=84$ and 117) available around the SHiP target material one $(\mathcal{A}=96)$. Both give very similar results, with a cross section varying within $\pm 6 \%$ from the NNPDF2.3 proton PDF one, depending on the $\gamma^{\mathrm{D}}$ mass. The alternative generator Madgraph5_aMC@NLO v2.7.2 [48] is also used to cross check the cross section calculation. The parameterisation of the width of the resonance, dependent on the branching ratios to fermion pairs, is a little different and explains the difference seen.

$1.4<m_{\gamma^{\mathrm{D}}} \leq 3 \mathrm{GeV}: \sigma_{\mathrm{QCD}}=\varepsilon^{2} \times e^{-2.05488-1.96804 \times m_{\gamma} \mathrm{D}}$,

$m_{\gamma^{\mathrm{D}}}>3 \mathrm{GeV}: \quad \sigma_{\mathrm{QCD}}=\varepsilon^{2} \times e^{-5.51532-0.830917 \times m_{\gamma^{\mathrm{D}}} \mathrm{D}}$.

Higher-order contributions to the process could lead to a sizable increase of the cross section at such low masses. Using the MATRIX v1.0.5 program [49-54], the ratio of next-to-next-to-leading-order (NNLO) over LO differential cross sections for standard Drell-Yan production at $\sqrt{s}=$ $27.43 \mathrm{GeV}$ as a function of the dilepton invariant mass $\mathrm{M}_{\ell \ell}$ is found to be rather flat at $1.7 \pm 0.17$ (stat) for $\mathrm{M}_{\ell \ell}$ between 1.4 and $5 \mathrm{GeV}$, increasing up to 2.2 at $\mathrm{M}_{\ell \ell}=10 \mathrm{GeV}$. The QCD scale uncertainties on the ratio are found to $b_{-11 \%}^{+20 \%}$ in the range $2<\mathrm{M}_{\ell \ell}<5 \mathrm{GeV}$ increasing above $\pm 30 \%$ for $\mathrm{M}_{\ell \ell}$ above $10 \mathrm{GeV}$ or below $2 \mathrm{GeV}$. The MSTW $2008 \mathrm{NNLO}$ PDF set [55] has been used for all calculations. These calculations are also found to be in fair agreement (within 10\%) with a study performed more specifically in proton-antiproton collisions, with a special interest for low dilepton masses and in particular for $\sqrt{s}=30 \mathrm{GeV}$, from Ref. [56], and consider-

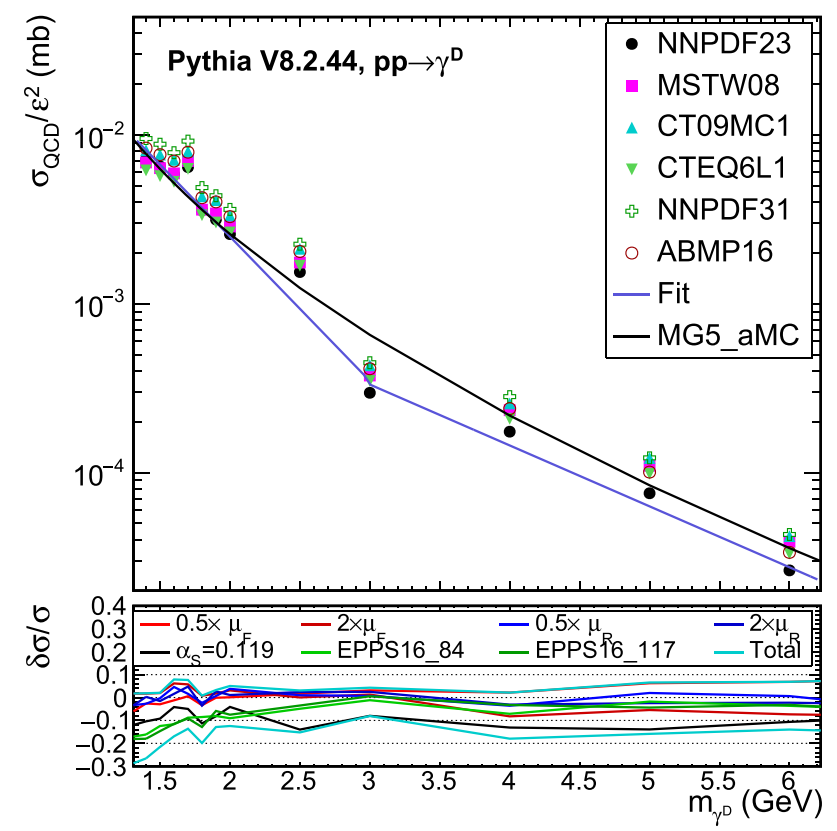

Fig. 5 QCD production cross section at LO as a function of $m_{\gamma^{\mathrm{D}}}$. The fit function is described in Eq. (9). The upper pad shows the impact of different PDF sets [55,57-60]. The lower pad shows the relative uncertainties from several theoretical uncertainty sources on the cross section calculated by PYTHIA, and their sum in quadrature under "Total"

ing soft-gluon resummations at all orders. Given the lack of experimental data at these low masses and low $\sqrt{s}$ to confirm the size of the expected correction and the impact from PDF and non-perturbative effects on the actual dark photon production, a final $\mathrm{k}$-factor of $1.7 \pm 0.7$ is applied to the $\mathrm{LO}$ PYTHIA cross section from Eq. (9).

The relative contribution from each process is shown in Fig. 6, as a function of $m_{\gamma}$ D, for the three production modes, in the two scenarios considered for the proton bremsstrahlung mode.

\subsection{Dark photon decays}

Except for the meson production mode, in which the new particle couples to the parent meson via mixing with the photon and hence cannot be a resonance from PYTHIA's point-of-view, in QCD and proton bremsstrahlung the $\gamma^{\mathrm{D}}$ is implemented as a resonance. In all cases, the decay channels are implemented as follows.

The partial decay width of the dark photon into a lepton pair is given by [36]:

$$
\begin{aligned}
\Gamma\left(\gamma^{\mathrm{D}} \rightarrow \ell^{+} \ell^{-}\right)= & \frac{1}{3} \alpha_{\mathrm{Q} E D} m_{\gamma^{\mathrm{D}} \varepsilon^{2}} \sqrt{1-\frac{4 m_{\ell}^{2}}{m_{\gamma^{\mathrm{D}}}^{2}}} \\
& \times\left(1+\frac{2 m_{\ell}^{2}}{m_{\gamma^{\mathrm{D}}}^{2}}\right),
\end{aligned}
$$



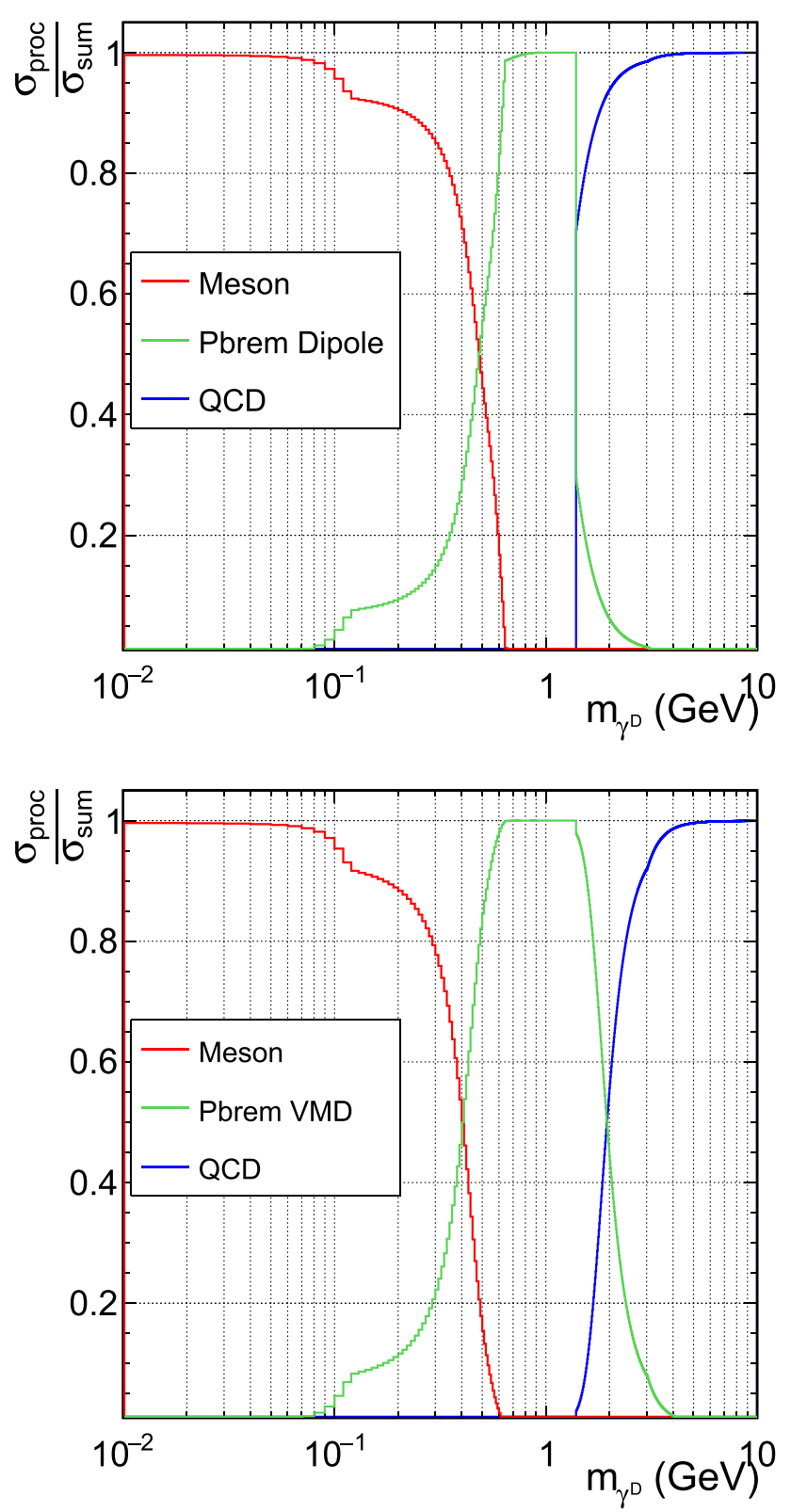

Fig. 6 Relative contributions to the cross section as a function of $m_{\gamma}$ D for the three production modes studied, using the dipole form factor for proton bremsstrahlung (top) or the VMD form factor (bottom)

where $m_{\ell}$ is the lepton mass, for electron, muon or tau leptons, if kinematically allowed. Following the approach used by the authors of Ref. [61], the partial decay width into quark pairs is computed as:

$\Gamma\left(\gamma^{\mathrm{D}} \rightarrow\right.$ hadrons $)=\Gamma\left(\gamma^{\mathrm{D}} \rightarrow \mu^{+} \mu^{-}\right) R\left(m_{\gamma^{\mathrm{D}}}\right)$,

where

$R(\sqrt{s})=\frac{\sigma\left(e^{+} e^{-} \rightarrow \text { hadrons }\right)}{\sigma\left(e^{+} e^{-} \rightarrow \mu^{+} \mu^{-}\right)}$,

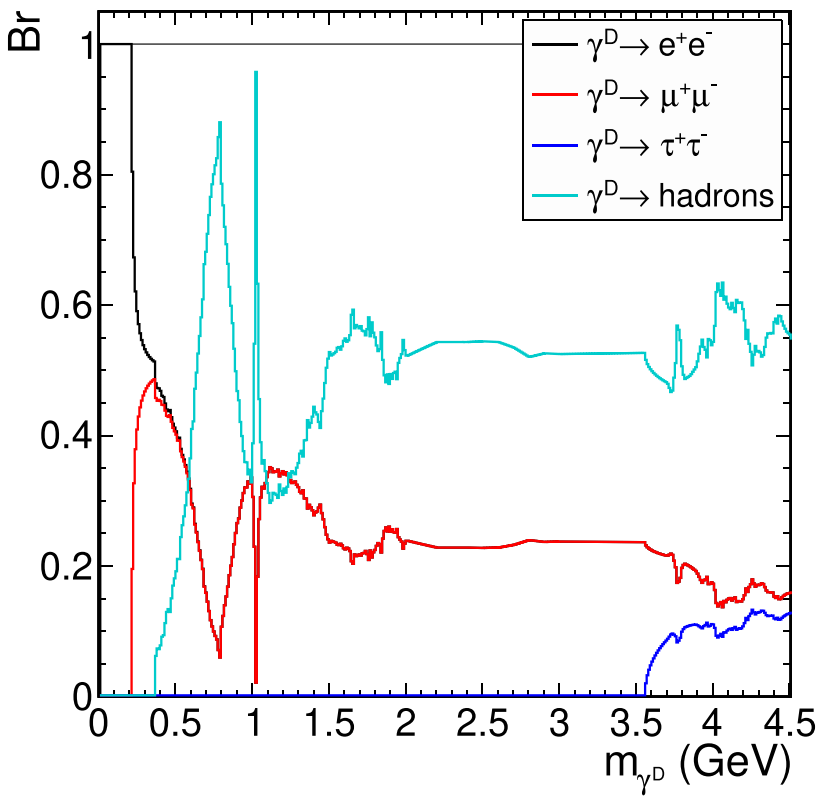

Fig. 7 Branching ratio of the $\gamma^{\mathrm{D}}$ into pairs of leptons or quarks as a function of its mass

is the energy-dependent R-ratio quantifying the hadronic annihilation in $e^{+} e^{-}$collisions [62], tabulated from 0.3 to $10.29 \mathrm{GeV}$.

The lifetime of the $\gamma^{\mathrm{D}}$ is then naturally set to the inverse of its total width, summing all the kinematically-allowed channels for calculating the total width. It is proportional to $1 / \varepsilon^{2}$. The branching ratios to individual channels are set to the ratio of the partial over total width, and are hence independent of $\varepsilon$. For separating the hadronic channels into the different quark-flavoured pairs allowed kinematically, the coupling is assumed to be proportional to the quark charge $q$ as $\mathrm{n}_{\mathcal{C}} \times q^{2}$ [63], with $\mathrm{n}_{\mathcal{C}}=3$ the number of coloured charges. When the $\gamma^{\mathrm{D}}$ is implemented as a resonance in PYTHIA, the decay goes explicitly through the pair of quarks, before hadronisation. Otherwise the hadrons are found as direct decay products of the $\gamma^{\mathrm{D}}$.

The branching ratio of the $\gamma^{\mathrm{D}}$ into pairs of leptons or quarks is shown in Fig. 7 as a function of $m_{\gamma^{D}}$. The hadronic decays become available above the pion mass threshold. The expected lifetime of the $\gamma^{\mathrm{D}}$ as a function of its mass and $\varepsilon$ mixing parameter is shown in Fig. 8.

\section{SHiP sensitivity}

In order to maximise the statistical power of the limited number of events produced with PYTHIA in the different production modes, the $\gamma^{\mathrm{D}}$ decay vertex position is randomly assigned to be inside the decay vessel of length $\mathrm{LVessel}=$ $50.760 \mathrm{~m}$, and the associated probability of this happening is given as a function of the $\gamma^{\mathrm{D}}$ four-momentum $\left(\mathrm{p}, \mathrm{E}_{\gamma^{\mathrm{D}}}\right)$ and 


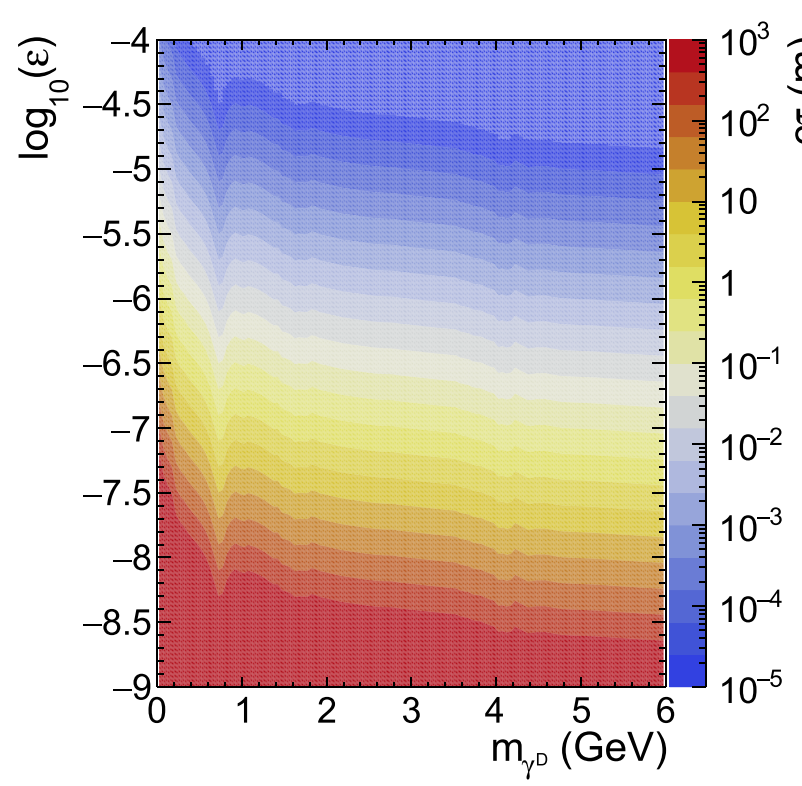

Fig. 8 Expected lifetime of the dark photon as a function of its mass and of the kinetic mixing parameter $\varepsilon$

lifetime $\mathrm{c} \tau$ :

$\mathrm{W}_{\mathrm{vtx}}(\ell)=e^{-\frac{\ell+\mathrm{L}_{0}}{\beta \times \gamma \times \mathrm{c \tau}}} \times \frac{\mathrm{L}_{\text {Vessel }}}{\beta \times \gamma \times \mathrm{c} \tau}$,

with $\gamma=E_{\gamma^{\mathrm{D}}} / \sqrt{E_{\gamma^{\mathrm{D}}}^{2}-p^{2}}, \beta=p / E_{\gamma^{\mathrm{D}}}, \mathrm{L}_{0}$ is the distance from the target to the entrance of the decay vessel, and $\ell$ is randomly distributed between 0 and $\mathrm{L}_{\text {Vessel }}$ with a flat prior.

The total event rate expected is then extracted from the cross sections $\sigma_{\text {prod }}$ defined in Sect. 3, i.e. Eqs. (4), (8) and (9) for the meson, proton bremsstrahlung and QCD productions, respectively, normalising to the luminosity expected from the $\mathrm{N}=2 \times 10^{20}$ proton-on-target events that will be collected by the end of the SHiP physics program. The expected rate is taking into account the detector acceptance and the efficiency to reconstruct the decay products in the SHiP detector, $\mathcal{P}_{\text {vessel }}$ and $\mathcal{P}_{\text {reco }}$ described in detail in Sects. 4.2 and 4.3, respectively, and following Eq. (14):

$\begin{aligned} \mathcal{N}_{\gamma^{\mathrm{D}}}= & \sigma_{\text {prod }} \times \mathcal{L}_{\mathrm{SHiP}} \times \operatorname{Br}\left(\gamma^{\mathrm{D}} \rightarrow \mathrm{ch}+\mathrm{ch}\right) \\ & \times \mathcal{P}_{\text {vessel }} \times \mathcal{P}_{\text {reco }} .\end{aligned}$

The SHiP luminosity is defined as: $\mathcal{L}_{\mathrm{SHiP}}=\frac{\mathrm{N}}{\sigma_{\text {SHiP }}^{\text {inel }}}$, using an inelastic proton-nucleon cross section of $\sigma_{\mathrm{SHiP}}^{\text {inel }}=$ $10.7 \mathrm{mb}$ [15], which directly corresponds to the SHiP target material (Molybdenum) nuclear interaction length and density.

The strategy of the analysis relies on identifying the decays of the $\gamma^{\mathrm{D}}$ into at least two charged particles, $\gamma^{\mathrm{D}} \rightarrow \mathrm{ch}+$ ch. The reconstructed charged tracks must originate from a common vertex. These requirements are enough to ensure that almost no background event will survive the selection,

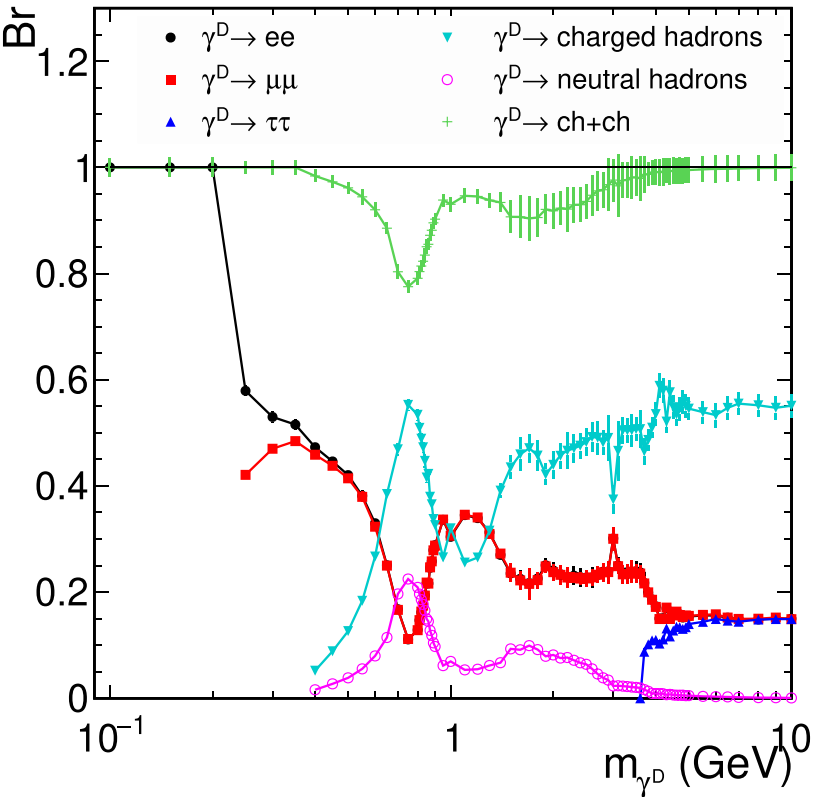

Fig. 9 Branching ratio to the visible decay channels, as a function of $m_{\gamma^{\mathrm{D}}} \cdot \operatorname{Br}\left(\gamma^{\mathrm{D}} \rightarrow \mathrm{ch}+\mathrm{ch}\right)$ is equal to $1-\operatorname{Br}\left(\gamma^{\mathrm{D}} \rightarrow\right.$ neutral hadrons $)$

as demonstrated in Refs. [5,15]. The $90 \%$ confidence level (CL) limits on the existence of a $\gamma^{\mathrm{D}}$ with given $\left(m_{\gamma^{\mathrm{D}}}, \varepsilon\right)$ are hence set by excluding regions where more than $\mathcal{N}_{\gamma^{\mathrm{D}}}=2.3$ events are expected.

\subsection{Decay channels}

The following final states are considered, whenever available for a given $m_{\gamma^{\mathrm{D}}}: \mathrm{e}^{+} \mathrm{e}^{-}, \mu^{+} \mu^{-}, \tau^{+} \tau^{-}$, and any hadronic decay channels leading to charged particles (e.g. $\pi^{+} \pi^{-}+X$, $\left.K^{+} K^{-}+X\right)$. The branching ratio to the different final states is shown in Fig. 9 for all the simulated $\left(m_{\gamma^{\mathrm{D}}}, \varepsilon\right)$ points in the three different production modes, as a function of $m_{\gamma}$, calculating the mean value over the different $\varepsilon$ samples. All events classified under " $\mathrm{e}^{+} \mathrm{e}^{-}$", " $\mu^{+} \mu^{-}$", " $\tau^{+} \tau^{-}$" and "charged hadrons" have at least two charged particles, their sum is represented as "ch + ch". Only the events classified under "neutral hadrons" are lost due to the analysis selection described in Sect. 4.3. Compared to Fig. 7, Fig. 9 highlights the mass scan actually simulated, and the separation of the hadronic final states into the charged and neutral ones.

\subsection{Vessel acceptance}

For events which have two charged particles, the $\gamma^{\mathrm{D}}$ decay vertex is further required to be within the vessel volume. The efficiency of this selection, $\mathcal{P}_{\text {vessel }}$ is defined as the ratio of the sum of the weights $\mathrm{w}_{\mathrm{vtx}}(\ell)$ of events passing the vertex selection described in Table 2 over the total number of events with a dark photon decaying to at least two charged particles. 
This efficiency is shown in Fig. 10 as a function of $\left(m_{\gamma^{\mathrm{D}}}, \varepsilon\right)$, for the three production modes. It is mostly driven by the lifetime of the $\gamma^{\mathrm{D}}$, and the kinematics of the $\gamma^{\mathrm{D}}$ produced in the target. Its maximum is around $5 \%$ for the production via meson decay, $10 \%$ for the proton bremsstrahlung production, and for higher masses in QCD production.

\subsection{Reconstruction of the decay products}

The strategy employed in this analysis relies uniquely on the reconstruction of charged particles by the SHiP straw tracker. Future extensions of this work could consider also calorimeter deposits (with the possibility to fully reconstruct $\pi^{0}$ decays to two photons) and muon detectors. Events are retained if two tracks are found passing the criteria summarised in Table 2, namely that the two tracks are within the fiducial area of the detector up to the fourth layer after the magnet, the fit converged with good quality requirements $\left(\chi^{2} / \mathrm{NDF}<5\right.$ with NDF the number of degrees of freedom of the fit). The tracks are required to have an impact parameter (IP) less than $0.1 \mathrm{~m}$ in the $(\mathrm{x}, \mathrm{y})$ plane, a momentum $p$ above $1 \mathrm{GeV}$, and a distance of closest approach (DOCA) below $1 \mathrm{~cm}$. Criteria on the number of hits (NDF $>25)$ or presence of hits before/after the magnet are meant to reduce backgrounds which could come from particles re-entering the detector volume due to the magnetic field. At the moment, the resolution of the timing detector is neglected, and MC truth information is used instead.

The efficiency of having two good tracks passing the selection for events which had two charged particles and $\gamma^{\mathrm{D}}$ vertex in the decay volume, $\mathcal{P}_{\text {reco }}$ is shown in Fig. 11. Once the $\gamma^{\mathrm{D}}$ decays in the vessel volume, the reconstruction efficiency is above $80 \%$ in most of the parameter space. For production via meson decay, a dependency on $\varepsilon$ is observed, with the efficiency dropping to below $50 \%$ as $\varepsilon$ decreases. This is found to be related to the wider angular distribution of dark photons produced in meson decays, introducing a dependency on the position of the decay vertex.

\subsection{Systematic uncertainties}

The following sources of systematic uncertainties from theory are investigated, for the three production modes. The missing contributions from cascade decays will be the subject of future work and is not considered.

For the meson production, the overall rate is affected by the following uncertainties:

- Branching ratios of the mesons to decay channels with photons from Table 1: from Ref. [37], the uncertainties on the measurement of these branching ratios are 0.03 , $0.5,3.4$ and $3.6 \%$ for $\pi^{0} \rightarrow \gamma \gamma, \eta^{0} \rightarrow \gamma \gamma, \omega \rightarrow \pi^{0} \gamma$ and $\eta^{\prime} \rightarrow \gamma \gamma$ respectively, translating directly to the final rate.

- Uncertainty on the meson multiplicities and shape of their kinematics properties: PYTHIA 8.2 has been compared with data in several existing publications. A comparison to NA27 and NA56 data is made in Ref. [64] for the inclusive production of $\pi^{0}$ mesons, and reasonable agreement is found, within $30 \%$ in the kinematic regions targeted by our measurement. In this comparison, the Pythia $\mathrm{p}-\mathrm{p}$ cross section is scaled by a factor $\mathrm{A}^{2 / 3}$ to be compared to the $\mathrm{p}$-Be collisions from the data. The scaled Pythia cross section is also found to be in good agreement with the full parametrisation of the pion invariant cross section taken from Ref. [65]. PHENIX and ALICE also measured inclusive $\pi^{0}, \eta$ and $\omega$ production and ratios [6668], and showed global agreement within about $20 \%$ with the PYTHIA 8 (Monash 2013 Tune) simulation.

Adding the different sources in quadrature, this results in a total systematic uncertainty of $\pm 30 \%$.

For the proton bremsstrahlung, the theory systematic uncertainties concern:

- uncertainties on the inelastic p-p cross section $\sigma_{p p}(s)$, which will mostly cancel in the ratio $\frac{\sigma_{p p}\left(s^{\prime}\right)}{\sigma_{p p}(s)}$, are neglected.

- Dipole form factor versus VMD form factor: the two scenarios are presented separately in the final exclusion limits.

- Contribution from protons undergoing elastic scattering before radiating the $\gamma^{\mathrm{D}}$ : an upper bound is derived using a factor $\frac{1}{1-P_{\mathrm{el}}}=1.34$ [69], with $P_{\mathrm{el}}$ the probability for an incoming proton to generate an elastic scattering, $P_{\mathrm{el}}=$ $\frac{\sigma_{\mathrm{pp}}^{\text {elastic }}}{\sigma_{\mathrm{pp}}^{\text {tot }}}$ and $\sigma_{\mathrm{pp}}^{\text {elastic }}=10.35 \mathrm{mb}$ from PYTHIA, summing elastic and single-diffractive contributions.

- Boundary conditions used in the integration of Eq. (8): by varying the upper bound on $p_{\perp}$ by $\pm 2 \mathrm{GeV}$, the total rate is changed by ${ }_{-30 \%}^{+15 \%}$. Varying the lower and upper bounds $p_{\min }\left(p_{\max }\right)$ by $\pm 0.04( \pm 0.04)$, the total rate is changed by ${ }_{-25 \%}^{+40 \%}$.

A total systematic uncertainty of ${ }_{-40 \%}^{+50 \%}$ is assumed to cover these sources.

For the QCD production, the theory systematic uncertainties concern the parameterisation of the LO cross section, the choice of NNLO k-factor and the impact from QCD scales and PDFs. Figure 5 shows the relative contributions from QCD scales and PDF on PYTHIA's LO cross section. The choice of PDF set is giving large variations in normalisation, but not affecting the overall shape of the cross section versus mass. The PDF set chosen is conservatively the one giving the lowest cross section. As discussed in Sect. 3.3, in the end the uncertainty is dominated by the NNLO k-factor of 

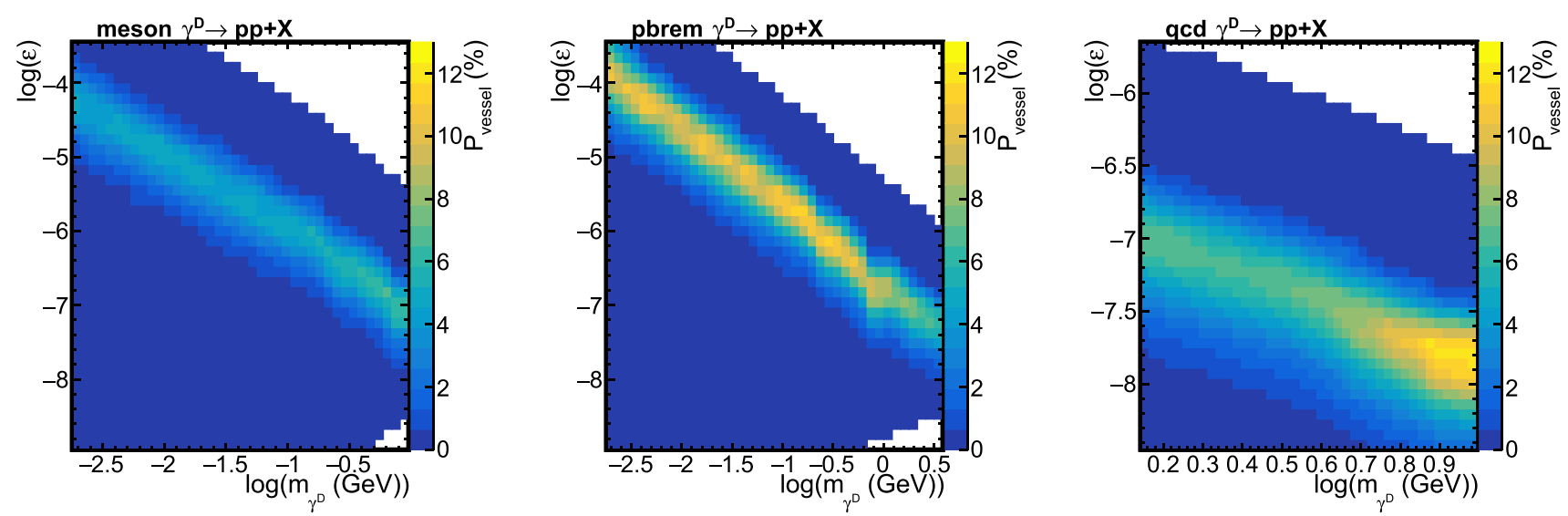

Fig. 10 Efficiency of requiring the $\gamma^{\mathrm{D}}$ decay vertex to be inside the decay vessel volume, for the three production modes studied. An interpolation between the simulated $\left(m_{\gamma^{\mathrm{D}}}, \varepsilon\right)$ values is performed using a linear interpolation via Delaunay triangulation

Table 2 Selection criteria applied on the reconstructed events. See Fig. 1 for the layout simulated

\begin{tabular}{ll}
\hline Decay vertex & $\mathrm{z}$ position within the range $[610,5076] \mathrm{cm}$ \\
& $\mathrm{x}-\mathrm{y}$ within vessel volume and at least $5 \mathrm{~cm}$ away from its inner walls \\
& In each layer - before and after magnet - up to tracking station 4 \\
Straw tracker hits & $\geq 2$ tracks \\
Tracks & $\mathrm{NDF}>25, \chi^{2} / \mathrm{NDF}<5, \mathrm{DOCA}<1 \mathrm{~cm}, \mathrm{p}>1 \mathrm{GeV}, \mathrm{IP}<0.1 \mathrm{~m}$ \\
\end{tabular}
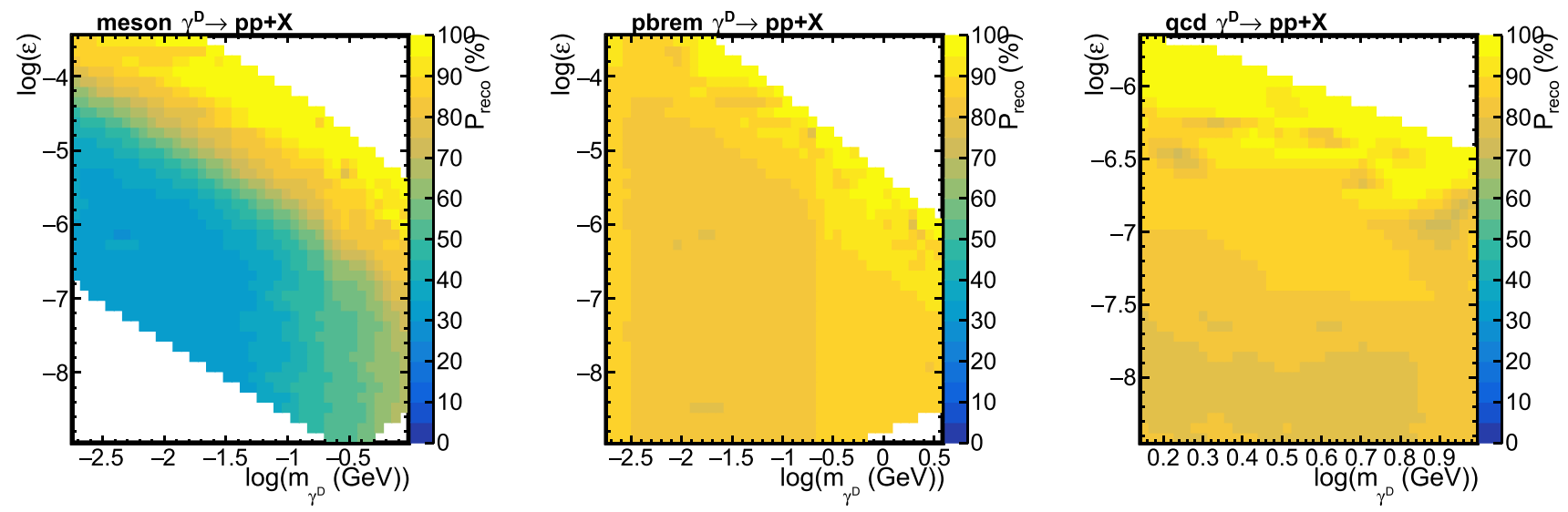

Fig. 11 Efficiency of requiring two good tracks, for events with two charged particles and $\gamma^{\mathrm{D}}$ vertex inside the vessel volume, for the three production modes studied. An interpolation between the simulated $\left(m_{\gamma} \mathrm{D}, \varepsilon\right)$ values is performed using a linear interpolation via Delaunay triangulation

$1.7 \pm 0.7$. The total systematic uncertainty is hence taken as $\pm 40 \%$.

Experimental systematic uncertainties concern the measurement of the luminosity, the modeling of the tracking efficiency and the assumptions entering the 0-background estimate. They have been neglected in this study, as they are expected to be small compared to the theoretical uncertainties.

\subsection{Extraction of the limit}

Events are generated following a discrete grid in $\left(m_{\gamma^{D}}, \varepsilon\right)$ values, and passed through the full simulation of the SHiP detector and reconstruction algorithms. The $\gamma^{\mathrm{D}}$ mass is varied between the electron-pair production threshold and $10 \mathrm{GeV}$, in 0.001 to $1 \mathrm{GeV}$ steps. The kinetic mixing parameter $\varepsilon$ is varied between $10^{-4}$ and $10^{-9}$ in varying-size steps in $\log (\varepsilon)$.

To find the $\varepsilon$ values that allow to reach 2.3 expected events, the expected rate is studied as a function of $\varepsilon$ for the discrete mass points, with a linear interpolation between fullysimulated values. Between mass points, a linear interpola- 

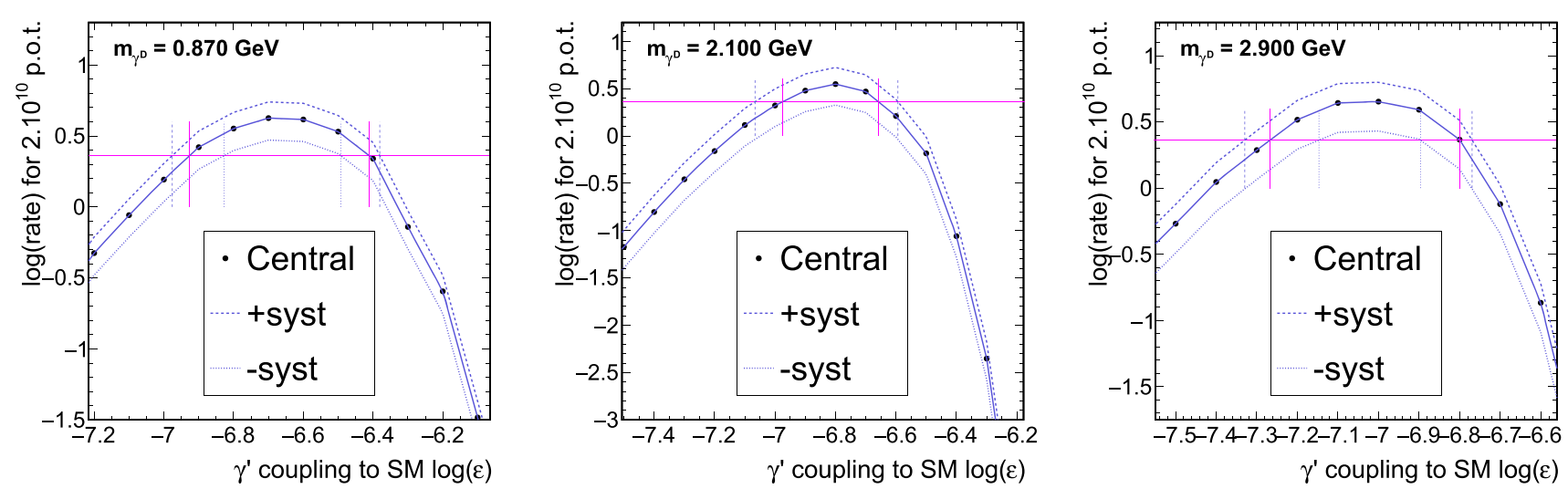

Fig. 12 Expected rate as a function of $\varepsilon$, for $m_{\gamma^{D}}=0.87$ (left), 2.1 (middle) or 2.9 (right) $\mathrm{GeV}$ and meson, proton bremsstrahlung with dipole form factor or QCD production, respectively. The horizontal pink line shows the 2.3 events threshold used to set the limit. Vertical

pink (dashed blue) lines show the result of the interpolation (varying the yields up and down according to the total systematic uncertainties detailed in Sect. 4.4)

Fig. 13 Expected $90 \%$ exclusion region as a function of the dark photon mass and of the kinetic mixing parameter $\varepsilon^{2}$, for the three production modes studied (top), and their combinations for the two proton bremsstrahlung scenarios (bottom). The dashed lines highlight the 1- $\sigma$ uncertainty band using the systematics described in Sect. 4.4. The excluded region in grey is from Ref. [70]
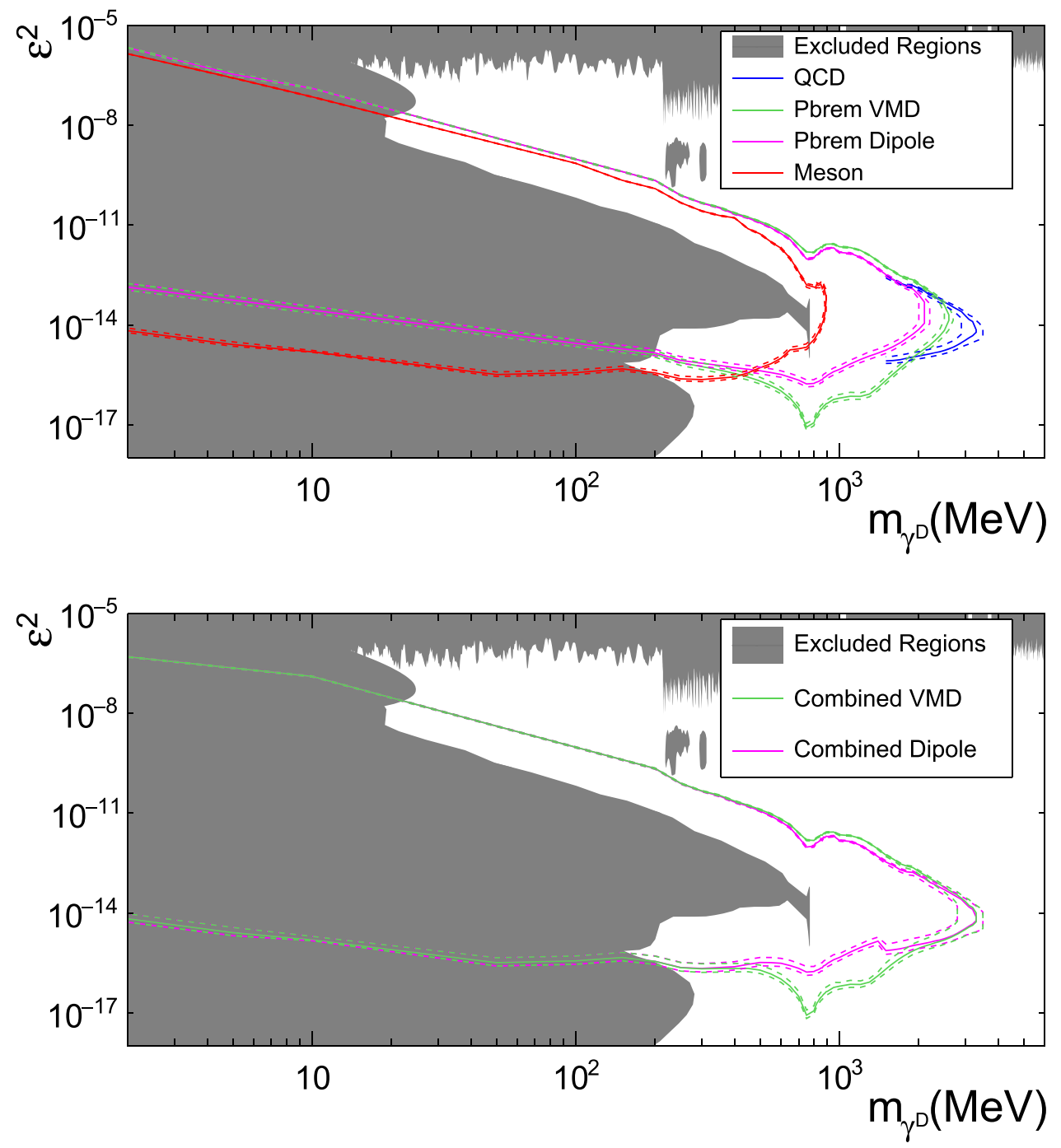
tion is also performed. The rate of events is driven by two aspects. For large $\varepsilon$ values, larger cross sections are expected but the detection efficiency decreases rapidly due to small lifetimes and decays happening before the decay vessel. As $\varepsilon$ decreases, the cross section decreases as $\varepsilon^{2}$ but the events have more and more probability to reach the vessel and the rate increases, up to a turning point where the decay vertex happens after the decay vessel and/or the cross section becomes too small. Hence the $90 \%$ CL exclusion region is contained inside a lower and upper limits on $\varepsilon^{2}$ for each mass point. The dependency of the excluded region on the mass is driven by the kinematic properties of the $\gamma^{\mathrm{D}}$ and its decay products, affecting the detector acceptance and selection efficiency.

As shown in Fig. 12 for representative mass points, for all processes, the upper bounds have little dependency on the absolute normalisation of the rate (so in particular systematic uncertainties on the cross sections and other quantities affecting the overall rate), due to the very steep dependency of the rate as a function of $\varepsilon$. The lower bounds are however more sensitive.

The 90\% CL exclusion contour is shown in Fig. 13 for the three production modes studied, and their combinations, in the $\left(m_{\gamma} \mathrm{D}, \varepsilon^{2}\right)$ plane. The excluded region shown in grey is from past experiments sensitive to this process [70]. For $\varepsilon^{2}>10^{-6}$, in the full mass range studied, the current sensitivity is coming from searches for dilepton resonances (e.g. Refs [71-78]). These results are complemented for low masses at lower $\varepsilon^{2}$ values by those from the reinterpretation of data from fixed-target experiments (e.g. [79-83]), and by recent dedicated searches for long-lived $\gamma^{\mathrm{D}}$ decaying to leptons $[84,85]$. The very-low coupling exclusions are from cosmological constraints, in particular bounds from Supernova 1987A data [86-88].

The SHiP experiment is expected to have a unique sensitivity in the mass region $\mathrm{m}_{\gamma^{\mathrm{D}}}$ ranging between 0.8 and $3.3_{-0.5}^{+0.2} \mathrm{GeV}$, and $\varepsilon^{2}$ ranging between $10^{-11}$ and $10^{-17}$.

\section{Conclusion}

The sensitivity of the SHiP detector has been investigated for the simplest vector portal model, in which the only hiddensector particle connecting to SM particles is a dark photon. The model is fully parameterised by only two parameters, the mass of the dark photon $m_{\gamma}$ D and the kinetic mixing parameter $\varepsilon$. Three different production mechanisms have been investigated, namely the production via meson decays from non-diffractive proton-nucleon interactions, by proton bremsstrahlung and by QCD parton-parton interaction.

Different sources of systematic uncertainties have been considered, dominated by theory predictions on the cross section times branching ratios (meson decays), two scenarios of nuclear form factor (proton bremsstrahlung) and higher-order corrections (QCD scattering). Only the primary proton-nucleon interaction is taken into account, secondaries from hadronic interactions in cascade decays could lead to an improvement in the sensitivity and will be the object of future work. The dark photon is assumed to decay to pairs of leptons or quarks, and only decay channels producing at least two charged particles coming from a common vertex are used.

With the selection applied, backgrounds are neglected and 90\% CL exclusion contours are extracted and compared with those from past experiments. The SHiP detector is expected to have a unique sensitivity for $\mathrm{m}_{\gamma^{\mathrm{D}}}$ ranging between 0.8 and $3.3_{-0.5}^{+0.2} \mathrm{GeV}$, and $\varepsilon^{2}$ ranging between $10^{-11}$ and $10^{-17}$.

Acknowledgements The SHiP Collaboration wishes to thank the Castaldo company (Naples, Italy) for their contribution to the development studies of the decay vessel. The SHiP Collaboration acknowledges support from the following funding agencies: the TAEK of Turkey with Grant number 2018TAEK(CERN)-A5.H6.F2-17; the National Research Foundation of Korea with grant numbers 2018R1A2B2007757, 2018R1D1A3B07050649, 2018R1D1A1B07050701, 2017R1D1A1B 03036042，2017R1A6A3A01075752，2016R1A2B4012302， and 2016R1A6A3A11930680; the Fundação para a Ciência e a Tecnologia of Portugal with grant number CERN/FIS-PAR/0030/2017; the Russian Foundation for Basic Research (RFBR), with Grant number 1702-00607.

Data Availability Statement This manuscript has associated data in a data repository. [Authors' comment: Data available within the DarkCast repository [89].]

Open Access This article is licensed under a Creative Commons Attribution 4.0 International License, which permits use, sharing, adaptation, distribution and reproduction in any medium or format, as long as you give appropriate credit to the original author(s) and the source, provide a link to the Creative Commons licence, and indicate if changes were made. The images or other third party material in this article are included in the article's Creative Commons licence, unless indicated otherwise in a credit line to the material. If material is not included in the article's Creative Commons licence and your intended use is not permitted by statutory regulation or exceeds the permitted use, you will need to obtain permission directly from the copyright holder. To view a copy of this licence, visit http://creativecomm ons.org/licenses/by/4.0/.

Funded by $\mathrm{SCOAP}^{3}$.

\section{References}

1. L. Evans, P. Bryant, JINST 3, S08001 (2008). https://doi.org/10. 1088/1748-0221/3/08/S08001

2. G. Apollinari, I. Béjar Alonso, O. Brüning, M. Lamont, L. Rossi, High-luminosity large hadron collider (HL-LHC): preliminary design report. Technical Report. CERN-2015-005, FERMILABDESIGN-2015-02, CERN, Geneva (2015)

3. C. Ahdida et al., A beam dump facility (BDF) at CERN-the concept and a first radiological assessment. Technical Report. CERNPBC-CONF-2019-001, CERN, Geneva (2019). https://cds.cern. $\mathrm{ch} /$ record/2655435. Accessed 21 May 2021 
4. B. Batell, M. Pospelov, A. Ritz, Phys. Rev. D 80, 095024 (2009). https://doi.org/10.1103/PhysRevD.80.095024

5. S. Alekhin et al., Rep. Prog. Phys. 79(12), 124201 (2016). https:// doi.org/10.1088/0034-4885/79/12/124201

6. B. Patt, F. Wilczek, Higgs-field portal into hidden sectors. Technical Report. MIT-CTP-3745 (2006). https://arxiv.org/abs/hep-ph/ 0605188

7. D. O'Connell, M.J. Ramsey-Musolf, M.B. Wise, Phys. Rev. D 75, 037701 (2007). https://doi.org/10.1103/PhysRevD.75.037701

8. B. Holdom, Phys. Lett. 166B, 196 (1986). https://doi.org/10.1016/ 0370-2693(86)91377-8

9. M. Bauer, P. Foldenauer, J. Jaeckel, JHEP 18, 094 (2020). https:// doi.org/10.1007/JHEP07(2018)094

10. D. Gorbunov, M. Shaposhnikov, J. High Energy Phys. 2007(10), 015 (2007). https://doi.org/10.1088/1126-6708/2007/10/015

11. M. Aaboud et al., JHEP 06, 166 (2018). https://doi.org/10.1007/ JHEP06(2018) 166

12. M. Aaboud et al., JHEP 10, 182 (2017). https://doi.org/10.1007/ JHEP10(2017)182

13. A.M. Sirunyan et al., Phys. Lett. B 796, 131 (2019). https://doi. org/10.1016/j.physletb.2019.07.013

14. V. Khachatryan et al., Phys. Lett. B 768, 57 (2017). https://doi.org/ 10.1016/j.physletb.2017.02.010

15. M. Anelli et al., A facility to search for hidden particles (SHiP) at the CERN SPS. Technical Report. CERN-SPSC-2015016. SPSC-P-350, CERN, Geneva (2015). Technical Proposal. arXiv:1504.04956. https://cds.cern.ch/record/2007512. Accessed 21 May 2021

16. W. Bonivento et al., Proposal to search for heavy neutral leptons at the SPS. Technical Report. CERN-SPSC-2013-024. SPSC-EOI010, CERN, Geneva (2013). http://cds.cern.ch/record/1606085. Accessed 21 May 2021

17. C. Ahdida et al., JHEP 04, 077 (2019). https://doi.org/10.1007/ JHEP04(2019)077

18. C. Ahdida et al., Measurement of the muon flux for the SHiP experiment. Technical Report. CERN-EP-2020-006, CERN, Geneva (2020). https://arxiv.org/abs/2001.04784

19. A. Akmete et al., JINST 12(05), P05011 (2017). https://doi.org/ 10.1088/1748-0221/12/05/P05011

20. C. Ahdida et al., SHiP experiment-comprehensive design study report. Technical Report. CERN-SPSC-2019-049. SPSC-SR263, CERN, Geneva (2019). https://cds.cern.ch/record/2704147. Accessed 21 May 2021

21. A. Miano, A. Fiorillo, A. Salzano, A. Prota, R. Jacobsson, Arch. Civ. Mech. Eng. 21, 3 (2020). https://doi.org/10.1007/ s43452-020-00152-9

22. I. Bereziuk, O. Bezshyyko, M. Ferro-Luzzi, Initial design studies of the SHiP straw detector. Technical Report. CERN-SHiPNOTE-2015-001, CERN, Geneva (2015). https://cds.cern.ch/ record/2005286. Accessed 21 May 2021

23. E. Van Herwijnen, H. Dijkstra, M. Ferro-Luzzi, T. Ruf, Simulation and pattern recognition for the SHiP Spectrometer Tracker. Technical Report. CERN-SHiP-NOTE-2015-002, CERN, Geneva (2015). https://cds.cern.ch/record/2005715. Accessed 21 May 2021

24. B. Hosseini, W.M. Bonivento, Particle identification tools and performance in the SHiP experiment. Technical Report. CERNSHiP-NOTE-2017-002, CERN, Geneva (2017). https://cds.cern. $\mathrm{ch} /$ record/2282039. Accessed 21 May 2021

25. T. Sjöstrand, S. Ask, J.R. Christiansen, R. Corke, N. Desai, P. Ilten, S. Mrenna, S. Prestel, C.O. Rasmussen, P.Z. Skands, Comput. Phys. Commun. 191, 159 (2015). https://doi.org/10.1016/j.cpc.2015.01. 024

26. C. Andreopoulos et al., Nucl. Instrum. Methods A614, 87 (2010). https://doi.org/10.1016/j.nima.2009.12.009

27. T. Sjöstrand, S. Mrenna, P. Skands, JHEP 5, 26. https://doi.org/10. 1088/1126-6708/2006/05/026
28. H. Dijkstra, T. Ruf, Heavy flavour cascade production in a beam dump. Technical Report. CERN-SHiP-NOTE-2015-009, CERN, Geneva (2015). https://cds.cern.ch/record/2115534. Accessed 21 May 2021

29. S. Agostinelli et al., Nucl. Instrum. Methods A506, 250 (2003). https://doi.org/10.1016/S0168-9002(03)01368-8

30. M. Al-Turany, D. Bertini, R. Karabowicz, D. Kresan, P. Malzacher, T. Stockmanns, F. Uhlig, J. Phys.: Conf. Ser. 396(2), 022001 (2012). https://doi.org/10.1088/1742-9596/396/2/022001

31. D. Gorbunov, A. Makarov, I. Timiryasov, Phys. Rev. D 91(3), 035027 (2015). https://doi.org/10.1103/PhysRevD.91.035027

32. R.D. Ball et al., Nucl. Phys. B 867, 244 (2013). https://doi.org/10. 1016/j.nuclphysb.2012.10.003

33. P. Skands, S. Carrazza, J. Rojo, Eur. Phys. J. C 74(8), 3024 (2014). https://doi.org/10.1140/epjc/s10052-014-3024-y

34. A. Berlin, S. Gori, P. Schuster, N. Toro, Phys. Rev. D 98(3), 035011 (2018). https://doi.org/10.1103/PhysRevD.98.035011

35. M. Aguilar-Benitez et al., Z. Phys. C 50, 405 (1991). https://doi. org/10.1007/BF01551452

36. J. Blümlein, J. Brunner, Phys. Lett. B 731, 320 (2014). https://doi. org/10.1016/j.physletb.2014.02.029

37. C. Patrignani et al., Chin. Phys. C 40(10), 100001 (2016). https:// doi.org/10.1088/1674-1137/40/10/100001

38. L.N. Hand, D.G. Miller, R. Wilson, Rev. Mod. Phys. 35, 335 (1963). https://doi.org/10.1103/RevModPhys.35.335

39. P. deNiverville, C.Y. Chen, M. Pospelov, A. Ritz, Phys. Rev. D 95(3), 035006 (2017). https://doi.org/10.1103/PhysRevD.95. 035006

40. A. Faessler, M.I. Krivoruchenko, B.V. Martemyanov, Phys. Rev. C 82, 038201 (2010). https://doi.org/10.1103/PhysRevC.82.038201

41. K. Nakamura et al., J. Phys. G: Nucl. Part. Phys. 37(7A), 075021 (2010). https://doi.org/10.1088/0954-3899/37/7a/075021

42. K. Kim, Y. Tsai, Phys. Rev. D 8(9), 3109 (1973)

43. K. Kim, Y. Tsai, Phys. Lett. B 40(6), 665 (1972). https://doi.org/ 10.1016/0370-2693(72)90622-3

44. L. Carloni, J. Rathsman, T. Sjöstrand, JHEP 04, 091 (2011). https:// doi.org/10.1007/JHEP04(2011)091

45. C. Ciobanu, T. Junk, G. Veramendi, J. Lee, G. De Lentdecker, K.S. McFarland, K. Maeshima, Z' generation with PYTHIA. Technical Report. FERMILAB-FN-0773-E, FERMILAB, Batavia (2005). https://doi.org/10.2172/15020136

46. A. Buckley, J. Ferrando, S. Lloyd, K. Nordström, B. Page, M. Rüfenacht, M. Schönherr, G. Watt, Eur. Phys. J. C 75, 132 (2015). https://doi.org/10.1140/epjc/s10052-015-3318-8

47. K.J. Eskola, P. Paakkinen, H. Paukkunen, C.A. Salgado, Eur. Phys. J. C 77(3), 163 (2017). https://doi.org/10.1140/epjc/ s10052-017-4725-9

48. J. Alwall, R. Frederix, S. Frixione, V. Hirschi, F. Maltoni, O. Mattelaer, H.S. Shao, T. Stelzer, P. Torrielli, M. Zaro, JHEP 07, 079 (2014). https://doi.org/10.1007/JHEP07(2014)079

49. M. Grazzini, S. Kallweit, M. Wiesemann, Eur. Phys. J. C 78(7), 537 (2018). https://doi.org/10.1140/epjc/s10052-018-5771-7

50. S. Catani, L. Cieri, G. Ferrera, D. de Florian, M. Grazzini, Phys. Rev. Lett. 103, 082001 (2009). https://doi.org/10.1103/ PhysRevLett.103.082001

51. F. Cascioli, P. Maierhöfer, S. Pozzorini, Phys. Rev. Lett. 108, 111601 (2012). https://doi.org/10.1103/PhysRevLett.108.111601

52. A. Denner, S. Dittmaier, L. Hofer, Comput. Phys. Commun. 212, 220 (2017). https://doi.org/10.1016/j.cpc.2016.10.013

53. S. Catani, L. Cieri, D. de Florian, G. Ferrera, M. Grazzini, Eur. Phys. J. C 72, 2195 (2012). https://doi.org/10.1140/epjc/ s10052-012-2195-7

54. S. Catani, M. Grazzini, Phys. Rev. Lett. 98, 222002 (2007). https:// doi.org/10.1103/PhysRevLett.98.222002

55. A.D. Martin, W.J. Stirling, R.S. Thorne, G. Watt, Eur. Phys. J. C 63, 189 (2009). https://doi.org/10.1140/epjc/s10052-009-1072-5 
56. H. Shimizu, G.F. Sterman, W. Vogelsang, H. Yokoya, Phys. Rev. D 71, 114007 (2005). https://doi.org/10.1103/PhysRevD.71.114007

57. R.D. Ball et al., Eur. Phys. J. C 77(10), 663 (2017). https://doi.org/ 10.1140/epjc/s10052-017-5199-5

58. H.L. Lai, J. Huston, S. Mrenna, P. Nadolsky, D. Stump, W.K. Tung, C.P. Yuan, JHEP 04, 035 (2010). https://doi.org/10.1007/ JHEP04(2010)035

59. J. Pumplin, D.R. Stump, J. Huston, H.L. Lai, P.M. Nadolsky, W.K. Tung, JHEP 07, 012 (2002). https://doi.org/10.1088/1126-6708/ 2002/07/012

60. S. Alekhin, J. Blümlein, S. Moch, R. Placakyte, Phys. Rev. D 96(1), 014011 (2017). https://doi.org/10.1103/PhysRevD.96.014011

61. J.D. Bjorken, R. Essig, P. Schuster, N. Toro, Phys. Rev. D 80, 075018 (2009). https://doi.org/10.1103/PhysRevD.80.075018

62. K.A. Olive et al., Chin. Phys. C 38, 090001 (2014). https://doi.org/ 10.1088/1674-1137/38/9/090001

63. M.X. Liu, Mod. Phys. Lett. A 32(10), 1730008 (2017). https://doi. org/10.1142/S0217732317300087

64. B. Döbrich, J. Jaeckel, T. Spadaro, JHEP 05, 213 (2019). https:// doi.org/10.1007/JHEP05(2019)213

65. M. Bonesini, A. Marchionni, F. Pietropaolo, T. Tabarelli de Fatis, Eur. Phys. J. C 20, 13 (2001). https://doi.org/10.1007/ s100520100656

66. S. Acharya et al., Eur. Phys. J. C 78(3), 263 (2018). https://doi.org/ 10.1140/epjc/s10052-018-5612-8

67. S. Acharya et al., Production of $\omega(782)$ in pp collisions at $\sqrt{s}$ $=7$ TeV. Technical Report. ALICE-PUBLIC-2018-004, CERN, Geneva (2018). http://cds.cern.ch/record/2316785. Accessed 21 May 2021

68. A. Adare et al., Phys. Rev. C 84, 044902 (2011). https://doi.org/ 10.1103/PhysRevC.84.044902

69. C. Ahdida et al., JHEP 4, 199 (2021). https://doi.org/10.1007/ JHEP04(2021)199

70. J. Beacham, C. Burrage, D. Curtin, A. De Roeck, J. Evans, J.L. Feng, C. Gatto, S. Gninenko, A. Hartin, I. Irastorza et al., J. Phys. G: Nucl. Part. Phys. 47(1), 010501 (2019). https://doi.org/10.1088/ $1361-6471 / \mathrm{ab} 4 \mathrm{~cd} 2$

71. J.P. Lees et al., Phys. Rev. Lett. 113, 201801 (2014). https://doi. org/10.1103/PhysRevLett.113.201801

72. J. Batley et al., Phys. Lett. B 746, 178 (2015). https://doi.org/10. 1016/j.physletb.2015.04.068

73. H. Merkel et al., Phys. Rev. Lett. 112, 221802 (2014). https://doi. org/10.1103/PhysRevLett.112.221802

74. F. Archilli et al., Phys. Lett. B 706(4), 251 (2012). https://doi.org/ 10.1016/j.physletb.2011.11.033
75. D. Babusci et al., Phys. Lett. B 720(1), 111 (2013). https://doi.org/ 10.1016/j.physletb.2013.01.067

76. D. Babusci et al., Phys. Lett. B 736, 459 (2014). https://doi.org/10. 1016/j.physletb.2014.08.005

77. A. Anastasi et al., Phys. Lett. B 757, 356 (2016). https://doi.org/ 10.1016/j.physletb.2016.04.019

78. R. Aaij et al., Phys. Rev. Lett. 120, 061801 (2018). https://doi.org/ 10.1103/PhysRevLett.120.061801

79. J.D. Bjorken, S. Ecklund, W.R. Nelson, A. Abashian, C. Church, B. Lu, L.W. Mo, T.A. Nunamaker, P. Rassmann, Phys. Rev. D 38, 3375 (1988). https://doi.org/10.1103/PhysRevD.38.3375

80. B. Batell, R. Essig, Z. Surujon, Phys. Rev. Lett. 113, 171802 (2014). https://doi.org/10.1103/PhysRevLett.113.171802

81. F. Bergsma et al., Phys. Lett. B 157(5), 458 (1985). https://doi.org/ 10.1016/0370-2693(85)90400-9

82. E.M. Riordan et al., Phys. Rev. Lett. 59, 755 (1987). https://doi. org/10.1103/PhysRevLett.59.755

83. A. Bross, M. Crisler, S. Pordes, J. Volk, S. Errede, J. Wrbanek, Phys. Rev. Lett. 67, 2942 (1991). https://doi.org/10.1103/PhysRevLett. 67.2942

84. R. Aaij et al., Phys. Rev. Lett. 124(4), 041801 (2020). https://doi. org/10.1103/PhysRevLett.124.041801

85. D. Banerjee et al., Phys. Rev. D 101(7), 071101 (2020). https://doi. org/10.1103/PhysRevD.101.071101

86. A. Fradette, M. Pospelov, J. Pradler, A. Ritz, Phys. Rev. D 90(3), 035022 (2014). https://doi.org/10.1103/PhysRevD.90.035022

87. D. Kazanas, R.N. Mohapatra, S. Nussinov, V.L. Teplitz, Y. Zhang, Nucl. Phys. B 890, 17 (2014). https://doi.org/10.1016/j.nuclphysb. 2014.11.009

88. J.H. Chang, R. Essig, S.D. McDermott, JHEP 01, 107 (2017). https://doi.org/10.1007/JHEP01(2017)107

89. P. Ilten, Y. Soreq, M. Williams, W. Xue, Serendipity in dark photon searches. JHEP 6, 4 (2018). https://doi.org/10.1007/ JHEP06(2018)004 


\section{SHiP Collaboration}

C. Ahdida ${ }^{44}$, A. Akmete ${ }^{48}$, R. Albanese ${ }^{14, \mathrm{~d}, \mathrm{~h}}$, A. Alexandrov ${ }^{14,32,34, \mathrm{~d}}$, A. Anokhina ${ }^{39, \mathrm{k}}$, S. Aoki ${ }^{18}$, G. Arduini ${ }^{44}$, E. Atkin ${ }^{38}$, N. Azorskiy ${ }^{29}$, J. J. Back ${ }^{54}$, A. Bagulya ${ }^{32}$, F. Baaltasar Dos Santos ${ }^{44}$, A. Baranov ${ }^{40}$, F. Bardou ${ }^{44}$, G. J. Barker ${ }^{54}$, M. Battistin ${ }^{44}$, J. Bauche ${ }^{44}$, A. Bay ${ }^{46}$, V. Bayliss ${ }^{51}$, G. Bencivenni ${ }^{15}$, A. Y. Berdnikov ${ }^{37}$, Y. A. Berdnikov ${ }^{37}$, M. Bertani ${ }^{15}$, C. Betancourt ${ }^{47}$, I. Bezshyiko ${ }^{47}$, O. Bezshyyko ${ }^{55}$, D. Bick ${ }^{8}$, S. Bieschke ${ }^{8}$, A. Blanco ${ }^{28}$, J. Boehm ${ }^{51}$, M. Bogomilov ${ }^{1}$, I. Boiarska ${ }^{3}$, K. Bondarenko 27,57, W. M. Bonivento ${ }^{13}$, J. Borburgh ${ }^{44}$, A. Boyarsky ${ }^{27,55}$, R. Brenner ${ }^{43}$, D. Breton ${ }^{4}$, V. Büscher ${ }^{10}$, A. Buonaura ${ }^{47}$, S. Buontempo ${ }^{14}$, S. Cadeddu ${ }^{13}$, A. Calcaterra ${ }^{15}$, M. Calviani ${ }^{44}$, M. Campanelli ${ }^{53}$, M. Casolino ${ }^{44}$, N. Charitonidis ${ }^{44}$, P. Chau ${ }^{10}$, J. Chauveau ${ }^{5}$, A. Chepurnov ${ }^{39}$, M. Chernyavskiy ${ }^{32}, \quad$ K.-Y. Choi ${ }^{26}$, A. Chumakov ${ }^{2}$, P. Ciambrone ${ }^{15}$, V. Cicero ${ }^{12}, \quad$ L. Congedo ${ }^{11, a}, \quad$ K. Cornelis ${ }^{44}, \quad$ M. Cristinziani ${ }^{7}, \quad$ A. Crupano ${ }^{14, d}$, G. M. Dallavalle ${ }^{12}$, A. Datwyler ${ }^{47}$, N. D’Ambrosio ${ }^{16}$, G. D'Appollonio ${ }^{13, c}$, R. de Asmundis ${ }^{14}$, J. De Carvalho Saraiva ${ }^{28}$, G. De Lellis ${ }^{14,34,44, d}$, M. de Magistris ${ }^{14, j}$, A. De Roeck ${ }^{44}$, M. De Serio ${ }^{11, a}$, D. De Simone ${ }^{47}$, L. Dedenko ${ }^{39}$, P. Dergachev ${ }^{34}$, A. Di Crescenzo 14,d, L. Di Giulio ${ }^{44}$, N. Di Marco ${ }^{16}$, C. Dib ${ }^{2}$ ， H. Dijkstra ${ }^{44}$, V. Dmitrenko ${ }^{38}$, L. A. Dougherty ${ }^{44}$, A. Dolmatov ${ }^{33}$, D. Domenici ${ }^{15}$, S. Donskov ${ }^{35}$, V. Drohan ${ }^{55}$, A. Dubreuil ${ }^{45}$, O. Durhan ${ }^{48}$, M. Ehlert ${ }^{6}$, E. Elikkaya ${ }^{48}$, T. Enik ${ }^{29}$, A. Etenko ${ }^{33,38}$, F. Fabbri ${ }^{12}$, O. Fedin ${ }^{36}$, F. Fedotovs ${ }^{52}$, G. Felici ${ }^{15}$, M. Ferrillo ${ }^{47}$, M. Ferro-Luzzi ${ }^{44}$, K. Filippov ${ }^{38}$, R. A. Fini ${ }^{11}$, P. Fonte ${ }^{28}$, C. Franco ${ }^{28}$, M. Fraser ${ }^{44}$, R. Fresa ${ }^{14, i, h}$, R. Froeschl ${ }^{44}$, T. Fukuda ${ }^{19}$, G. Galati ${ }^{14, d}$, J. Gall ${ }^{44}$, L. Gatignon ${ }^{44}$, G. Gavrilov ${ }^{36}$, V. Gentile ${ }^{14, \mathrm{~d}}$, B. Goddard ${ }^{44}$, L. Golinka-Bezshyyko55, A. Golovatiuk ${ }^{54, \mathrm{~d}}$, V. Golovtsov ${ }^{36}$, D. Golubkov ${ }^{30}$, A. Golutvin ${ }^{34,52}$, P. Gorbounov ${ }^{44}$, D. Gorbunov ${ }^{31}$, S. Gorbunov ${ }^{32}$, V. Gorkavenko ${ }^{55}$, M. Gorshenkov ${ }^{34}$, V. Grachev ${ }^{38}$, A. L. Grandchamp ${ }^{46}$, E. Graverini ${ }^{46}$, J.-L. Grenard ${ }^{44}$, D. Grenier ${ }^{44}$, V. Grichine ${ }^{32}$, N. Gruzinskii ${ }^{36}$, A. M. Guler ${ }^{48}$, Yu. Guz ${ }^{35}$, G. J. Haefeli ${ }^{46}$, C. Hagner ${ }^{8}$, H. Hakobyan ${ }^{2}$, I. W. Harris ${ }^{46}$, E. van Herwijnen ${ }^{34}$, C. Hessler ${ }^{44}$, A. Hollnage ${ }^{10}$, B. Hosseini ${ }^{52}$, M. Hushchyn ${ }^{40}$, G. Iasellii ${ }^{11, a}$, A. Iuliano ${ }^{14, d}$, R. Jacobsson ${ }^{44}$, D. Jokovićc ${ }^{41}$, M. Jonker ${ }^{44}$, I. Kadenko ${ }^{55}$, V. Kain ${ }^{44}$, B. Kaiser ${ }^{8}$, C. Kamiscioglu ${ }^{49}$, D. Karpenkov ${ }^{34}$, K. Kershaw ${ }^{44}$, M. Khabibullin ${ }^{31}$, E. Khalikov ${ }^{39}$, G. Khaustov ${ }^{35}$, G. Khoriauli ${ }^{10}$, A. Khotyantsev ${ }^{31}$, Y. G. Kim²3, V. Kim³,37, N. Kitagawa ${ }^{19}$, J.-W. Ko ${ }^{22}$, K. Kodama ${ }^{17}$, A. Kolesnikov ${ }^{29}$, D. I. Kolev ${ }^{1}$, V. Kolosov ${ }^{35}$, M. Komatsu ${ }^{19}$, A. Kono ${ }^{21}$, N. Konovalova ${ }^{32,34}$, S. Kormannshaus ${ }^{10}$, I. Korol $^{6}$, I. Korol'ko ${ }^{30}$, A. Korzenev ${ }^{45}$, V. Kostyukhin ${ }^{7}$, E. Koukovini Platia ${ }^{44}$, S. Kovalenko ${ }^{2}$, I. Krasilnikova ${ }^{34}$, Y. Kudenko ${ }^{31,38, g}$, E. Kurbatov ${ }^{40}$, P. Kurbatov ${ }^{34}$, V. Kurochka ${ }^{31}$, E. Kuznetsova ${ }^{36}$, H. M. Lacker ${ }^{6}$, M. Lamont ${ }^{44}$, G. Lanfranchi ${ }^{15}$, O. Lantwin ${ }^{34,47}$, A. Lauria ${ }^{14, \mathrm{~d}}$, K. S. Lee ${ }^{25}$, K. Y. Lee ${ }^{22}$, N. Leonardo ${ }^{28}$, J.-M. Lévy ${ }^{5}$, V. P. Loschiavo ${ }^{14, h}$, L. Lopes ${ }^{28}$, E. Lopez Sola ${ }^{44}$, V. Lyubovitskij ${ }^{2}$, J. Maalmi ${ }^{4}$, A.-M. Magnan ${ }^{52}$ (D), V. Maleev ${ }^{36}$, A. Malinin ${ }^{33}$, Y. Manabe ${ }^{19}$,

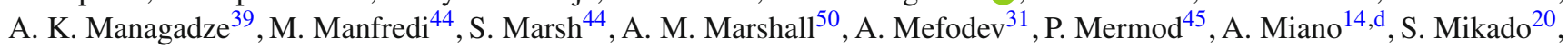
Yu. Mikhaylov ${ }^{35}$, D. A. Milstead ${ }^{42}$, O. Mineev ${ }^{31}$, A. Montanari ${ }^{12}$, M. C. Montesi ${ }^{14, d}$, K. Morishima ${ }^{19}$, S. Movchan ${ }^{29}$, Y. Muttoni ${ }^{44}$, N. Naganawa ${ }^{19}$, M. Nakamura ${ }^{19}$, T. Nakano ${ }^{19}$, S. Nasybulin ${ }^{36}$, P. Ninin ${ }^{44}$, A. Nishio ${ }^{19}$, B. Obinyakov ${ }^{33}$, $\begin{array}{llll}\text { S. Ogawa } & 21\end{array}$ N. Okateva $a^{32,34}$, B. Opitz ${ }^{8}$, J. Osborne ${ }^{44}, \quad$ M. Ovchynnikov ${ }^{27,55}$, N. Owtscharenko ${ }^{7}, \quad$ P. H. Owen ${ }^{47}$, P. Pacholek ${ }^{44}$, A. Paoloni ${ }^{15}$, B. D. Park ${ }^{22}$, A. Pastore ${ }^{11}$, M. Patel $^{34,52}$, D. Pereyma ${ }^{30}$, A. Perillo-Marcone ${ }^{44}$, G. L. Petkov ${ }^{1}$, K. Petridis ${ }^{50}$, A. Petrov ${ }^{33}$, D. Podgrudkov ${ }^{39, k}$, V. Poliakov ${ }^{35}$, N. Polukhina ${ }^{32,34,38}$, J. Prieto Prieto ${ }^{44}$, M. Prokudin ${ }^{30}$, A. Prota ${ }^{14, d}$, A. Quercia ${ }^{14, d}$, A. Rademakers ${ }^{44}$, A. Rakai ${ }^{44}$, F. Ratnikov ${ }^{40}$, T. Rawlings ${ }^{51}$, F. Redi46, S. Ricciardi ${ }^{51}$, M. Rinaldesi ${ }^{44}$, Volodymyr Rodin ${ }^{55}$, Viktor Rodin ${ }^{55}$, P. Robbe ${ }^{4}$, A. B. Rodrigues Cavalcante ${ }^{46}, \quad$ T. Roganova ${ }^{39}$, H. Rokujo ${ }^{19}$, G. Rosa ${ }^{14, d}$, T. Rovelli ${ }^{12, b}$, O. Ruchayskiy ${ }^{3}$, T. Ruf ${ }^{44}$, V. Samoylenko ${ }^{35}$, V. Samsonov ${ }^{38}$, F. Sanchez Galan ${ }^{44}$, P. Santos Diaz ${ }^{44}$, A. Sanz Ull ${ }^{44}$, A. Saputi ${ }^{15}$, O. Sato ${ }^{19}$, E. S. Savchenko ${ }^{34}$, J. S. Schliwinski ${ }^{6}$, W. Schmidt-Parzefall ${ }^{8}$, N. Serra ${ }^{34,47}$, S. Sgobba ${ }^{44}$, O. Shadura ${ }^{55}$, A. Shakin ${ }^{34}$, M. Shaposhnikov ${ }^{46}$, P. Shatalov ${ }^{30,34}$, T. Shchedrina ${ }^{32,34}$, L. Shchutska ${ }^{46}$, V. Shevchenko ${ }^{33,34}$, H. Shibuya ${ }^{21}$, S. Shirobokov ${ }^{52}$, A. Shustov ${ }^{38}$, S. B. Silverstein ${ }^{42}$, S. Simone ${ }^{11, a}$, R. Simoniello ${ }^{10}$, M. Skorokhvatov ${ }^{33,38}$, S. Smirnov ${ }^{38}$, G. Soares ${ }^{28}$, J. Y. Sohn ${ }^{22}$, A. Sokolenko ${ }^{55}$, E. Solodko ${ }^{44}$, N. Starkov ${ }^{32,34}$, L. Stoel ${ }^{44}$, M. E. Stramaglia ${ }^{46}$, D. Sukhonos ${ }^{44}$, Y. Suzuki ${ }^{19}$, S. Takahashi ${ }^{18}$, J. L. Tastet ${ }^{3}$, P. Teterin ${ }^{38}$, S. Than Naing ${ }^{32}$, I. Timiryasov ${ }^{46}$, V. Tioukov ${ }^{14}$, D. Tommasini ${ }^{44}$, M. Torii ${ }^{19}$, N. Tosi ${ }^{12}$, D. Treille ${ }^{44}$, R. Tsenov ${ }^{1,29}$, S. Ulin ${ }^{38}$, E. Ursov ${ }^{39, k}$, A. Ustyuzhanin ${ }^{40,34}$, Z. Uteshev ${ }^{38}$, L. Uvarov ${ }^{36}$, G. Vankova-Kirilova ${ }^{1}$, F. Vannucci ${ }^{5}$, V. Venturi ${ }^{44}$, I. Vidulin ${ }^{39, \mathrm{k}}, \mathrm{S}$. Vilchinski ${ }^{55}$, Heinz Vincke ${ }^{44}$, Helmut Vincke ${ }^{44}$, C. Visone ${ }^{14, \mathrm{~d}}, \mathrm{~K}$. Vlasik ${ }^{38}$, A. Volkov ${ }^{32,33}$, R. Voronkov $^{32}$, S. van Waasen ${ }^{9}$, R. Wanke ${ }^{10}$, P. Wertelaers ${ }^{44}$, O. Williams ${ }^{44}$, J.-K. Woo $^{24}$, M. Wurm ${ }^{10}$, S. Xella ${ }^{3}$, D. Yilmaz ${ }^{49}$, A. U. Yilmazer ${ }^{49}$, C. S. Yoon ${ }^{22}$, Yu. Zaytsev ${ }^{30}$, A. Zelenov ${ }^{36}$, J. Zimmerman ${ }^{6}$

\footnotetext{
${ }^{1}$ Faculty of Physics, Sofia University, Sofia, Bulgaria

${ }^{2}$ Universidad Técnica Federico Santa María and Centro Científico Tecnológico de Valparaíso, Valparaiso, Chile

${ }^{3}$ Niels Bohr Institute, University of Copenhagen, Copenhagen, Denmark

${ }^{4}$ LAL, Univ. Paris-Sud, CNRS/IN2P3, Université Paris-Saclay, Orsay, France

${ }^{5}$ LPNHE, IN2P3/CNRS, Sorbonne Université, Université Paris Diderot, 75252 Paris, France
} 
${ }^{6}$ Humboldt-Universität zu Berlin, Berlin, Germany

${ }^{7}$ Physikalisches Institut, Universität Bonn, Bonn, Germany

${ }^{8}$ Universität Hamburg, Hamburg, Germany

${ }^{9}$ Forschungszentrum Jülich GmbH (KFA), Jülich, Germany

${ }^{10}$ Institut für Physik and PRISMA Cluster of Excellence, Johannes Gutenberg Universität Mainz, Mainz, Germany

11 Sezione INFN di Bari, Bari, Italy

12 Sezione INFN di Bologna, Bologna, Italy

13 Sezione INFN di Cagliari, Cagliari, Italy

14 Sezione INFN di Napoli, Naples, Italy

15 Laboratori Nazionali dell' INFN di Frascati, Frascati, Italy

${ }^{16}$ Laboratori Nazionali dell'INFN di Gran Sasso, L'Aquila, Italy

17 Aichi University of Education, Kariya, Japan

18 Kobe University, Kobe, Japan

${ }^{19}$ Nagoya University, Nagoya, Japan

${ }^{20}$ College of Industrial Technology, Nihon University, Narashino, Japan

21 Toho University, Funabashi, Chiba, Japan

22 Physics Education Department and RINS, Gyeongsang National University, Jinju, Korea

${ }^{23}$ Gwangju National University of Education ${ }^{\mathrm{e}}$, Gwangju, Korea

24 Jeju National University ${ }^{\mathrm{e}}$, Jeju, Korea

25 Korea University, Seoul, Korea

26 Sungkyunkwan Universitye , Suwon-si, Gyeong Gi-do, Korea

27 University of Leiden, Leiden, The Netherlands

${ }^{28}$ LIP, Laboratory of Instrumentation and Experimental Particle Physics, Lisbon, Portugal

${ }^{29}$ Joint Institute for Nuclear Research (JINR), Dubna, Russia

${ }^{30}$ Institute of Theoretical and Experimental Physics (ITEP) NRC "Kurchatov Institute", Moscow, Russia

${ }^{31}$ Institute for Nuclear Research of the Russian Academy of Sciences (INR RAS), Moscow, Russia

32 P.N. Lebedev Physical Institute (LPI RAS), Moscow, Russia

33 National Research Centre "Kurchatov Institute", Moscow, Russia

34 National University of Science and Technology "MISiS", Moscow, Russia

35 Institute for High Energy Physics (IHEP) NRC "Kurchatov Institute", Protvino, Russia

36 Petersburg Nuclear Physics Institute (PNPI) NRC "Kurchatov Institute”, Gatchina, Russia

${ }^{37}$ St. Petersburg Polytechnic University (SPbPU) ${ }^{\mathrm{f}}$, St. Petersburg, Russia

38 National Research Nuclear University (MEPhI), Moscow, Russia

39 Skobeltsyn Institute of Nuclear Physics of Moscow State University (SINP MSU), Moscow, Russia

${ }^{40}$ Yandex School of Data Analysis, Moscow, Russia

${ }^{41}$ Institute of Physics, University of Belgrade, Belgrade, Serbia

42 Stockholm University, Stockholm, Sweden

43 Uppsala University, Uppsala, Sweden

${ }^{44}$ European Organization for Nuclear Research (CERN), Geneva, Switzerland

${ }^{45}$ University of Geneva, Geneva, Switzerland

${ }^{46}$ École Polytechnique Fédérale de Lausanne (EPFL), Lausanne, Switzerland

${ }^{47}$ Physik-Institut, Universität Zürich, Zurich, Switzerland

48 Middle East Technical University (METU), Ankara, Turkey

${ }^{49}$ Ankara University, Ankara, Turkey

${ }^{50}$ H.H. Wills Physics Laboratory, University of Bristol, Bristol, UK

51 STFC Rutherford Appleton Laboratory, Didcot, UK

52 Imperial College London, London, UK

53 University College London, London, UK

${ }^{54}$ University of Warwick, Warwick, UK

55 Taras Shevchenko National University of Kyiv, Kiev, Ukraine

a Università di Bari, Bari, Italy

b Università di Bologna, Bologna, Italy 
${ }^{\mathrm{c}}$ Università di Cagliari, Cagliari, Italy

d Università di Napoli “Federico II", Naples, Italy

e Associated to Gyeongsang National University, Jinju, Korea

f Associated to Petersburg Nuclear Physics Institute (PNPI), Gatchina, Russia

g Also at Moscow Institute of Physics and Technology (MIPT), Moscow Region, Russia

h Consorzio CREATE, Naples, Italy

${ }^{\mathrm{i}}$ Università della Basilicata, Potenza, Italy

j Università di Napoli Parthenope, Naples, Italy

k Also at Faculty of Physics M.V. Lomonosov Moscow State University, Moscow, Russia 